\title{
Uncertainty Quantification and Certification Prediction of Low-Boom Supersonic Aircraft Configurations
}

\author{
Thomas K. West IV* \\ Missouri University of Science and Technology, Rolla, MO, 65409 \\ Bryan W. Reuter ${ }^{\dagger}$ \\ University of Texas at Austin, Austin, TX, 78712 \\ Eric L. Walker ${ }^{\ddagger}$ Bil Kleb ${ }^{\S}$ and Michael A. Park \\ NASA Langley Research Center, Hampton, VA, 23681
}

\begin{abstract}
The primary objective of this work was to develop and demonstrate a process for accurate and efficient uncertainty quantification and certification prediction of low-boom, supersonic, transport aircraft. High-fidelity computational fluid dynamics models of multiple low-boom configurations were investigated including the Lockheed Martin SEEB-ALR body of revolution, the NASA $69^{\circ}$ Delta Wing, and the Lockheed Martin 1021-01 configuration. A nonintrusive polynomial chaos surrogate modeling approach was used for reduced computational cost of propagating mixed, inherent (aleatory) and model-form (epistemic) uncertainty from both the computation fluid dynamics model and the near-field to ground level propagation model. A methodology has also been introduced to quantify the plausibility of a design to pass a certification under uncertainty. Results of this study include the analysis of each of the three configurations of interest under inviscid and fully turbulent flow assumptions. A comparison of the uncertainty outputs and sensitivity analyses between the configurations is also given. The results of this study illustrate the flexibility and robustness of the developed framework as a tool for uncertainty quantification and certification prediction of low-boom, supersonic aircraft.
\end{abstract}

\section{Nomenclature}

$N_{s} \quad$ Number of Samples

$N_{t} \quad$ Number of Terms in a Total-Order

Polynomial Chaos Expansion

$n \quad$ Number of Random Dimensions

$p \quad$ Polynomial Order

$\alpha^{*} \quad$ Stochastic Response
$\Psi \quad$ Random Basis Function

$\xi \quad$ Standard Input Random Variable

$\alpha \quad$ Deterministic Coefficient in the Polynomial

Chaos Expansion

$C R \quad$ Confidence Ratio

\section{Introduction}

One of the most substantial obstacles to commercial supersonic flight over land is the creation of a sonic boom. Current Federal Aviation Administration and Committee on Aviation Environmental Protection restrictions prohibit commercial supersonic flight over many countries, including the United States, due to

\footnotetext{
*Graduate Student, Department of Aerospace and Mechanical Engineering, Student Member AIAA.

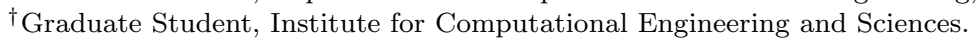

${ }^{\ddagger}$ Research Aerospace Engineer, Configuration Aerodynamics Branch, Senior Member AIAA.

$\S$ Assistant Branch Head, Computational AeroSciences Branch, Senior Member AIAA.

ๆResearch Scientist, Computational AeroSciences Branch, Senior Member AIAA.
} 
the ground level boom signature. Recent thrusts in research have focused on reducing the boom signature in hopes of reversing the regulations, in turn increasing the viability of supersonic flight. In 2003, after a series of tests that measured the ground signature of a F-5 Shaped Sonic Boom Demonstration (SSBD) supersonic aircraft, the SSBD program concluded sonic boom mitigation was possible through shape optimization. ${ }^{1}$ This has launched programs focusing on the design of low boom aircraft. Some examples are Gulfstream's Quiet Spike, Lockheed Martin's and Boeing's $N+1$ and $N+2$ supersonic designs, and Japan Aerospace Exploration Agency's (JAXA) NEXST and S3TD programs. ${ }^{2-5}$ In 2008, NASA held a Sonic Boom Prediction Workshop to assess the performance of computational fluid dynamics (CFD) for near-field boom prediction and a 2014 conference recently revisited this topic. ${ }^{6}$

As a means of aiding and accelerating the design process, CFD has become an important part of developing supersonic aircraft. Current, state of the art predictions use a three-dimensional CFD solvers to determine the near-field pressure signature, which is then numerically propagated to the ground level. These numerical results are often used to help inform the design of supersonic vehicles through various techniques, such as adjoint-based optimization employing near-field targeting or ground signature loudness ${ }^{7-9}$ and less computationally demanding derivative-free techniques. ${ }^{10}$ In any case, these computational models take one set of inputs (which may include the geometry, freestream conditions, angle-of-attack, or modeling parameters for turbulence and boom propagation) and produce a deterministic set of outputs (e.g., ground signature, final optimized geometry, and near-field flow information). Often, the input parameters may not be known exactly due to inherent variations in flight conditions and manufacturing tolerances. Additionally, error is introduced through assumptions made when creating the relevant mathematical models. Supersonic aircraft are sensitive to these changes and this motivates the development of a framework which can account for and efficiently represent the uncertainties in the design space for use in reliable and robust design of supersonic low-boom configurations that will meet necessary performance standards.

The primary objective of this study is to develop and demonstrate a framework for accurate and efficient uncertainty quantification (UQ) and certification prediction methodology for low-boom configurations. Because of the computational demand of low-boom configuration models, traditional sampling techniques, such as Monte Carlo, may not be feasible. The goal is to minimize the number of computationally expensive deterministic model evaluations needed for an accurate UQ analysis. In this study, a non-intrusive polynomial chaos surrogate modeling approach is used to represent the CFD and boom propagation models for each low-boom configuration investigated. Uncertainty in low-boom configuration modeling included mixed model-form (epistemic) and inherent (aleatory) uncertainty, which are propagated through the surrogate models. Three geometries were identified to be of interest and are used as demonstration configurations of the developed framework. The configurations selected were an axisymmetric, body of revolution (BOR) from Lockheed-Martin (SEEB), the NASA $69^{\circ}$ Delta Wing, and the Lockheed Martin (LM) 1021-01 low-boom aircraft configuration.

The following section of this paper introduces the computational modeling of sonic boom signatures, as well as how signatures predicted near the configuration are propagated to the ground. Section III then describes the UQ methodology employed, which included the creation of surrogate models for the output quantities of interest. A brief description of the types of uncertainty and the propagation of mixed uncertainty are given in Section IV. Section V describes the methodology utilized for certification prediction of selected performance metrics for low-boom configurations. The UQ and certification prediction framework is then summarized in Section VI and demonstrated in Section VII using three low-boom configurations. Important conclusions obtained from this study are summarized in the final section.

\section{Modeling and Propagation of Sonic Booms}

In this study, a high-fidelity approach was used to model sonic booms produced by low-boom configurations. This involved a high resolution CFD analysis near the body (the near-field). The near-field domain includes the vehicle and a region extending multiple body lengths away from the aircraft. The goal is to resolve the near-field pressure signature, parallel to the flow direction, that is generated by the body in supersonic flow. An example of this is shown in Figure 1. This signature is then propagated to ground level using a high fidelity propagation code called sBOOM. ${ }^{9}$ This simulates how the signal will change while passing through the atmosphere. Once a final ground signature is predicted, output quantities of interest, such as perceived loudness (PLdB) and C-weighted sound exposure level (CSEL), are evaluated and may serve as design and certification metrics. The remainder of this section gives further detail regarding the 
CFD simulations and the propagation model.

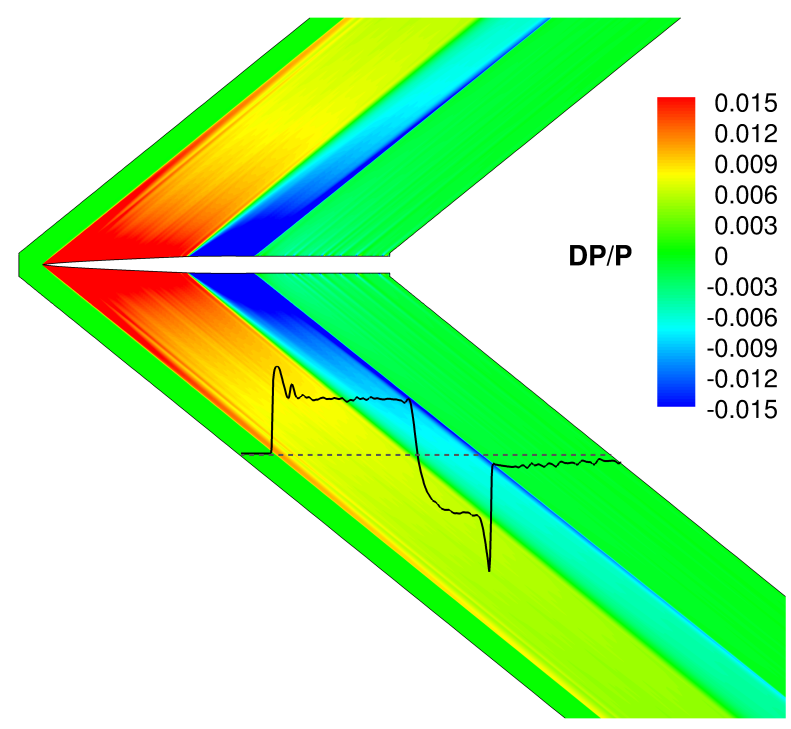

Figure 1: Near-Field CFD Domain with Pressure Signature

\section{A. Computational Fluid Dynamics Modeling}

This study employed the Fully Unstructured Navier-Stokes Three-Dimensional (FUN3D) ${ }^{11}$ flow solver for CFD analysis of low-boom configurations. FUN3D contains many tools for design optimization and analysis and was developed and is maintained at the NASA Langley Research Center. The code employs the finite volume formulation and stores flow field variables at the control volume nodes. FUN3D can handle aerodynamic simulations across a large range of Mach numbers from the subsonic to hypersonic regimes and has been used extensively for the analysis of sonic boom prediction. ${ }^{12-14}$

All cases investigated in this study were assumed to be at steady state. Often, the Euler equations are solved in place of the full Navier-Stokes equations for high Reynolds number, aerodynamic flows by assuming the flow is inviscid. While this is a known simplification of the actual flow physics, it allows for computational savings when investigating large-scale-complex problems or when large numbers of simulations are needed, such as when performing UQ. However, low-boom configurations may be designed to exploit viscous effects, which smear or dampen shocks. This would be overlooked when using an Euler assumption. In the current study, both inviscid and viscous, fully turbulent cases were explored. When solving the Euler equations, the inviscid fluxes were calculated at cell edges by employing the van Leer scheme to solve an approximate Riemann problem. For viscous cases, the inviscid fluxes are calculated by the Roe scheme for the approximate Riemann problem, if possible. In order to aid convergence, the viscous simulations of the LM-1021 and $69^{\circ}$ Delta wing required the use of the dissipative Low Diffusion Flux Splitting scheme. For modeling the turbulence, the one equation Spalart-Allmaras ${ }^{15}$ model was employed for computational efficiency and robustness.

\section{B. Computational Grids}

The configurations of interest (SEEB-ALR, 69 Delta Wing, and the LM 1021-01) are shown in Figure 2. The SEEB-ALR model is described by Morgenstern et al. ${ }^{3}$ and the $69^{\circ}$ Delta Wing is discussed by Hunton et al. ${ }^{16}$ The LM 1021-01 low-boom configuration is detailed by Morgenstern et al. ${ }^{17}$ The discussion of the physical models, their origins, and design specific details are left to these studies.

The inviscid SEEB-ALR and inviscid Delta Wing grids were the same grids made available for the 2014 AIAA Sonic Boom Prediction Workshop. The SEEB-ALR and Delta Wing models began as STEP files and a solid representation was extracted. Then, a triangular surface mesh and tetrahedral volume mesh were generated using GridEx. ${ }^{19}$ The core grid was then extruded using the Inflate method ${ }^{13}$ in a direction aligned 


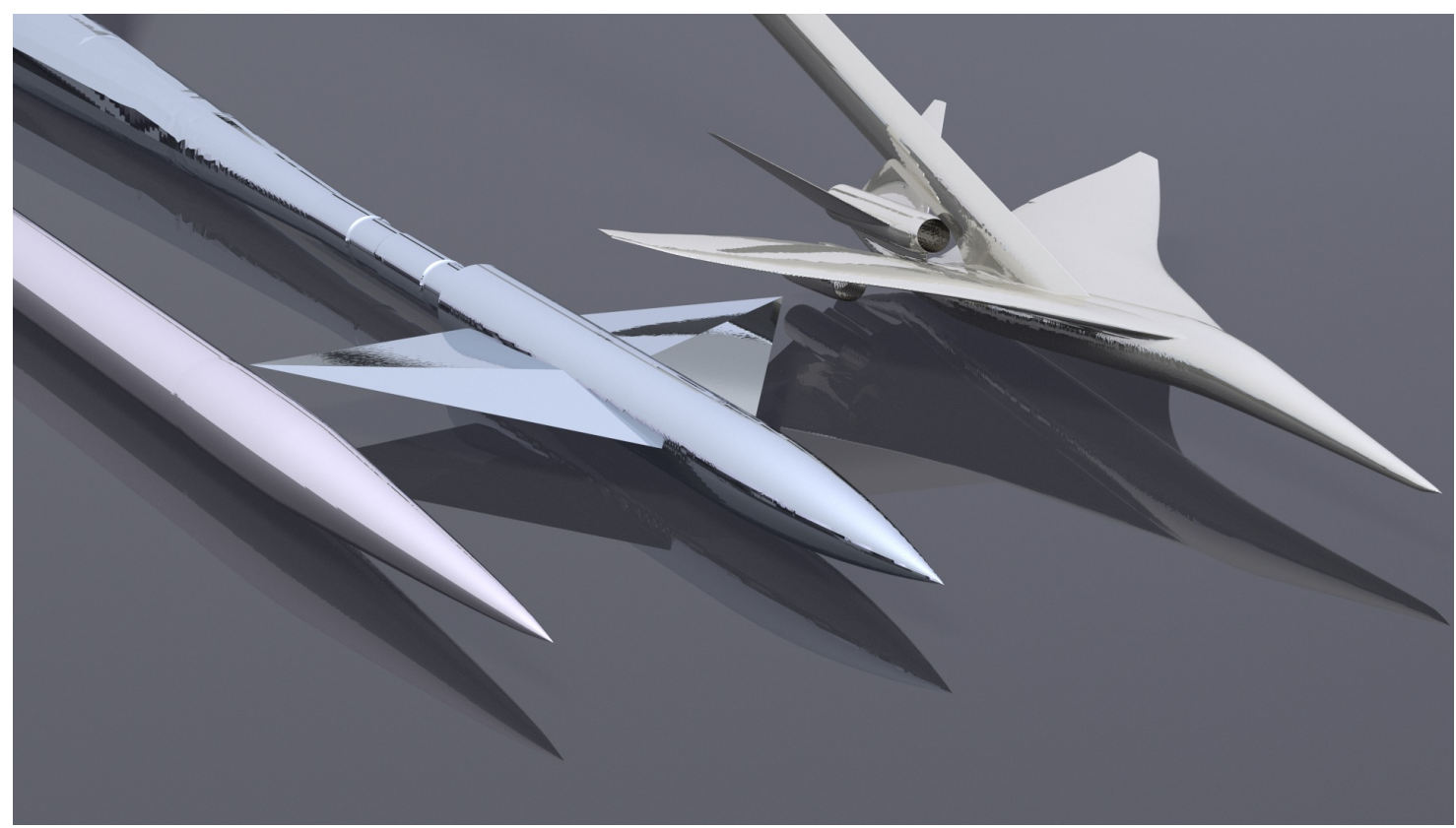

Figure 2: Configurations Studied in the Present Work. SEEB-ALR (left), NASA $69^{\circ}$ Delta Wing (center), LM 1020-01 (right). Models Not to Relative Scale. Reproduced with permission from Aftosmis et al. ${ }^{18}$

with the Mach angle so the relevant pressure signatures can be captured without wasting points in regions which are not influenced by the aircraft. Full details on the SEEB-ALR and $69^{\circ}$ Delta Wing solid models, grids, and grid generation techniques for near-field sonic boom CFD are given in Park et al. ${ }^{13} \mathrm{~A}$ cross section showing both the core grid and part of extruded region for the Delta Wing model is included in Figure 3.

One point of interest is that two versions of the SEEB-ALR solid model exist: the as-built and the as-designed. The as-designed geometry has a perfectly smooth surface, but the as-built model used in the wind tunnel testing possessed many surface imperfections as shown in Figure 4. It will later be shown that these flaws are reflected in the near-field signature. One objective of this study will be to quantify the effect of the surface imperfections on the loudness quantities of interest in this study.

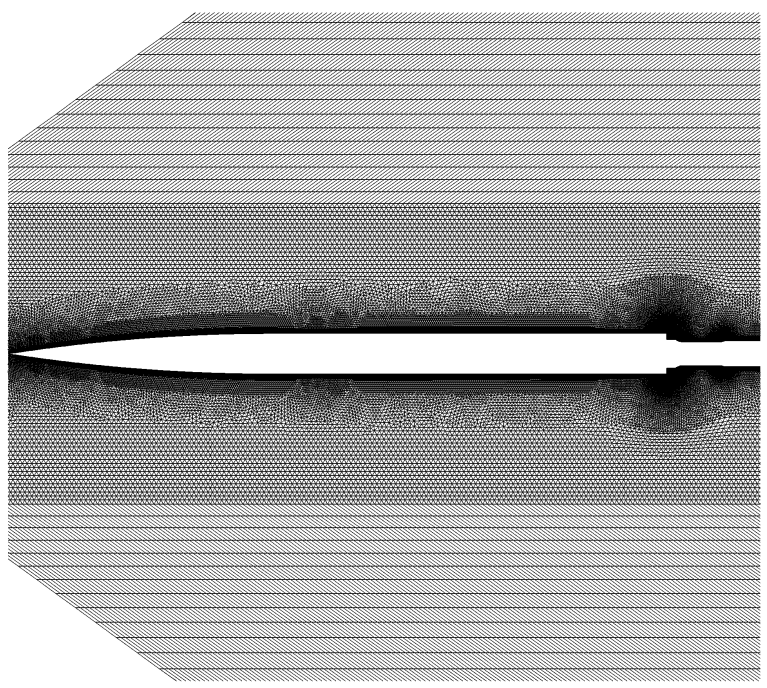

Figure 3: Computational Grid for the Viscous $69^{\circ}$ Delta Wing Including Extruded Region

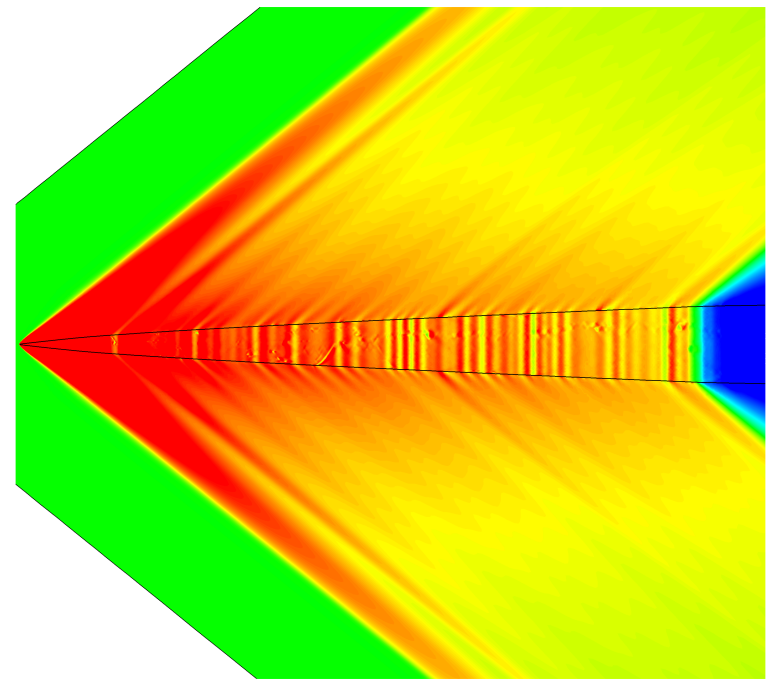

Figure 4: SEEB-ALR Surface Imperfections of the as-Built Model 
Table 1 summarizes the grid sizes for each model. In general, viscous grids needed to be much more refined. Coupled with the turbulence model, viscous cases took 5-10 times longer to converge than the Euler cases. This is especially significant for the LM-1021. All cases were run in parallel over 192 processors.

Table 1: CFD Grid Dimensions and Computational Time demands

\begin{tabular}{cccc}
\hline \hline Model & Cells & Nodes & Solution Time \\
\hline SEEB-ALR Euler & $7.83 \mathrm{e}+06$ & $2.89 \mathrm{e}+06$ & $20 \mathrm{~min}$ \\
SEEB-ALR Viscous & $3.64 \mathrm{e}+07$ & $6.20 \mathrm{e}+06$ & $1.3 \mathrm{hrs}$ \\
$69^{\circ}$ Delta Wing Euler & $2.24 \mathrm{e}+07$ & $5.36 \mathrm{e}+06$ & $10 \mathrm{~min}$ \\
$69^{\circ}$ Delta Wing Viscous & $7.80 \mathrm{e}+06$ & $2.90 \mathrm{e}+06$ & $2 \mathrm{hrs}$ \\
LM 1021-01 Euler & $3.38 \mathrm{e}+07$ & $8.37 \mathrm{e}+06$ & $30 \mathrm{~min}$ \\
LM 1021-01 Viscous & $7.24 \mathrm{e}+07$ & $2.45 \mathrm{e}+07$ & $5 \mathrm{hrs}$ \\
\hline \hline
\end{tabular}

To lessen the computational burden of the multiple CFD evaluations required for the UQ analysis, baseline cases with unperturbed parameter values were completed for each vehicle. These were then restarted for each random sample vector within the stochastic parameter space. For every configuration, this resulted in fewer iterations to achieve convergence compared to a completely new solution. This was especially significant for the LM-1021.

\section{Near-field Signature Propagation}

After obtaining the near-field pressure signature from the CFD model, it can then be propagated to the ground using a program called sBOOM. This model uses an augmented Burgers equation to propagate the near-field pressure signature to the ground level. The model takes into account nonlinear effects, thermoviscous absorption, atmospheric stratification, spreading, and many other molecular relaxation phenomena. Further details regarding this model are given by Rallabhandi. ${ }^{20}$

\section{Uncertainty Quantification Methodology}

Many high-fidelity numerical models, such as those discussed in section II, are computationally demanding. When performing UQ, traditional sampling methods may be problematic as large numbers of evaluations of the deterministic model are typically required for accurate results. In this study, a surrogate modeling approach based on polynomial chaos theory is utilized for improved computational efficiency. This section details the formulation of the surrogate models for use in accurate and efficient UQ.

\section{A. Point-Collocation Nonintrusive Polynomial Chaos}

In recent studies ${ }^{21-25}$ the polynomial chaos method has been used as a means of UQ over traditional methods, such as Monte Carlo, for its computational efficiency. Polynomial chaos is a surrogate modeling technique based on the spectral representation of the uncertainty. An important aspect of spectral representations is the decomposition of a response value or random function $\alpha^{*}$ into separable deterministic and stochastic components, as shown in Eq. (1).

$$
\alpha^{*}(\mathbf{x}, \boldsymbol{\xi}) \approx \sum_{i=0}^{P} \alpha_{i}(\mathbf{x}) \Psi_{i}(\boldsymbol{\xi})
$$

Here, $\alpha_{i}$ is the deterministic component and $\Psi_{i}$ is the random variable basis functions corresponding to the $\mathrm{i}^{\text {th }}$ mode. $\alpha^{*}$ is assumed to be a function of the vector $\mathbf{x}$ of independent random variables and the $n$-dimensional standard random variable vector $\boldsymbol{\xi}$. Note that this series is, by definition, an infinite series. However, in practice, it is truncated and a discrete sum is taken over a number of output modes. ${ }^{26}$ To form a complete basis or for a total order expansion, $N_{t}$ terms are required, which can be computed from Eq. (2) for a polynomial chaos expansion (PCE) of order $p$ and a number of random dimensions or variables, $n$. 


$$
N_{t}=P+1=\frac{(n+p) !}{n ! p !}
$$

Further details on polynomial chaos theory are given by Walters and Huyse, ${ }^{27}$ Eldred ${ }^{26}$ and Ghanem. ${ }^{28}$

The objective with any PCE method is to determine the expansion coefficients, $\alpha_{i}$. To do this, polynomial chaos methods can be implemented using an intrusive or a non-intrusive approach. While an intrusive method may appear straightforward in theory, for complex problems this process may be time consuming, expensive, and difficult to implement. ${ }^{21}$ In contrast, the non-intrusive approach can be easily implemented to construct a surrogate model that represents a complex computational simulation, because no modification to the deterministic model is required. The non-intrusive methods require only the response (or sensitivity) (29-31 $^{29}$ values at selected sample points to approximate the stochastic response surface.

Several methods have been developed for non-intrusive polynomial chaos (NIPC). Of these, the pointcollocation NIPC method has been used extensively in many aerospace simulations and CFD problems. ${ }^{22,23,25,29}$ The point-collocation method starts with replacing a stochastic response or random function with its PCE using Eq. (1). Then, $N_{t}$ vectors are chosen in random space and the deterministic code is then evaluated at these points, which is the left hand side of Eq. (1). Following this, a linear system of $N_{t}$ equations can be formulated and solved for the spectral modes of the random variables. This system is shown in Eq. (3).

$$
\left(\begin{array}{cccc}
\Psi_{0}\left(\boldsymbol{\xi}_{0}\right) & \Psi_{1}\left(\boldsymbol{\xi}_{0}\right) & \cdots & \Psi_{P}\left(\boldsymbol{\xi}_{0}\right) \\
\Psi_{0}\left(\boldsymbol{\xi}_{1}\right) & \Psi_{1}\left(\boldsymbol{\xi}_{1}\right) & \cdots & \Psi_{P}\left(\boldsymbol{\xi}_{1}\right) \\
\vdots & \vdots & \ddots & \vdots \\
\Psi_{0}\left(\boldsymbol{\xi}_{P}\right) & \Psi_{1}\left(\boldsymbol{\xi}_{P}\right) & \cdots & \Psi_{P}\left(\boldsymbol{\xi}_{P}\right)
\end{array}\right)\left(\begin{array}{c}
\alpha_{0} \\
\alpha_{1} \\
\vdots \\
\alpha_{P}
\end{array}\right)=\left(\begin{array}{c}
\alpha^{*}\left(\mathbf{x}, \boldsymbol{\xi}_{0}\right) \\
\alpha^{*}\left(\mathbf{x}, \boldsymbol{\xi}_{1}\right) \\
\vdots \\
\alpha^{*}\left(\mathbf{x}, \boldsymbol{\xi}_{P}\right)
\end{array}\right)
$$

Note that for this linear system, $N_{t}$ is the minimum number of deterministic samples required to obtain an analytical solution (i.e., the coefficient vector). If more samples are available and that are linearly independent, the system is considered overdetermined and can be solved using a least squares approach. The number of samples over the required minimum is represented by the use of an oversampling ratio (OSR), defined as the ratio of number of actual samples to the minimum number required (i.e., $N_{t}$ ). In general, the number of collocation points can be determined by multiplying Eq. (2) by an OSR. Hosder et al. ${ }^{32}$ determined an effective OSR of two for the stochastic model problems studied. It was shown that the accuracy of the PCE is dependent on the number of collocation points.

\section{Types of Uncertainty and Mixed Uncertainty Propagation}

A critical step in any uncertainty analysis is the classification of the uncertain parameters. These parameters may be mathematically represented differently based on the nature of their uncertainty. Incorrect classification and/or treatment of uncertain parameters can result in widely varying output uncertainty. The objective of this section is to describe the two main categories of uncertainty that exist in numerical modeling and outline a procedure for propagating uncertainty when both types are present.

\section{A. Types of Uncertainty}

Two main types of uncertainty exist in numerical modeling: aleatory uncertainty and epistemic uncertainty. ${ }^{33}$ Aleatory uncertainty is the inherent variation of a physical system. Such variation is due to the random nature of input data and can be mathematically represented by a probability density function if substantial experimental data is available for estimating the distribution type. An example of this for stochastic CFD simulations could be the fluctuation in freestream quantities. While still considered a random variable, these variables are not controllable and their uncertainty is sometimes referred to as irreducible.

Epistemic uncertainty in a stochastic problem comes from several potential sources. These include a lack of knowledge or incomplete information of the behavior of a particular variable. Also, ignorance or negligence with regards to accurate treatment of model parameters is a source of epistemic uncertainty. Contrary to aleatory uncertainty, epistemic uncertainty is sometimes referred to as reducible uncertainty. An increase in knowledge regarding the physics of a problem, along with accurate modeling, can reduce the amount of this type of uncertainty. Epistemic uncertainty is typically modeled using intervals because the use of probabilistic distributions (even a uniform distribution) can lead to inaccurate predictions in the amount of 
uncertainty in a system. Upper and lower bounds of these intervals can be drawn from limited experimental data or from expert predictions and judgment. ${ }^{21,22}$

An additional, special case of epistemic uncertainty is numerical error. This uncertainty is common in numerical modeling and is defined as a recognizable deficiency in any phase or activity of modeling and simulations that is not due to lack of knowledge of the physical system. In CFD, an example of this type of uncertainty would be the discretization error in both the temporal and spatial domains that comes from the numerical solution of the partial differential equations that govern the system. ${ }^{22}$ This uncertainty can be well understood and controlled through code verification and grid convergence studies.

\section{B. Mixed Uncertainty Quantification}

Many stochastic problems, including those to be used in this study, may contain both epistemic and aleatory types of uncertainty. The desired approach is to consider the contribution of both types of uncertainty simultaneously by propagating the mixed uncertainty through the stochastic model. This can be done using a procedure known as second-order probability. An NIPC response surface can be used within second-order probability in place of the deterministic code, as shown in Figure 5. Traditionally, second-order probability is a type of double loop sampling. In the outer loop, a vector of specific values for the epistemic variables is passed into the inner loop where the stochastic response surface resulting from the NIPC analysis is sampled for the single epistemic sample vector and every aleatory sample vector. The process is repeated for all of the epistemic sample vectors. This means that the total number of samples of the NIPC response surface is the number of epistemic samples times the number of aleatory samples. While many samples are typically required, In general, sampling the surrogate model is much less computationally expensive than sampling the deterministic model.

Each iteration of the outer loop generates a cumulative distribution function (CDF) based on the aleatory uncertainty analysis in the inner loop. After completion of the process, what remains is a series of CDFs, which, when plotted, gives intervals of the output variable from the model at different probability levels (i.e., a probability or "P-box" representation of mixed uncertainty output). Second-order probability can also be implemented with a Monte Carlo approach that uses the original model in the place of the response surface, which is done for the first model problem in this study for comparison with the NIPC results.

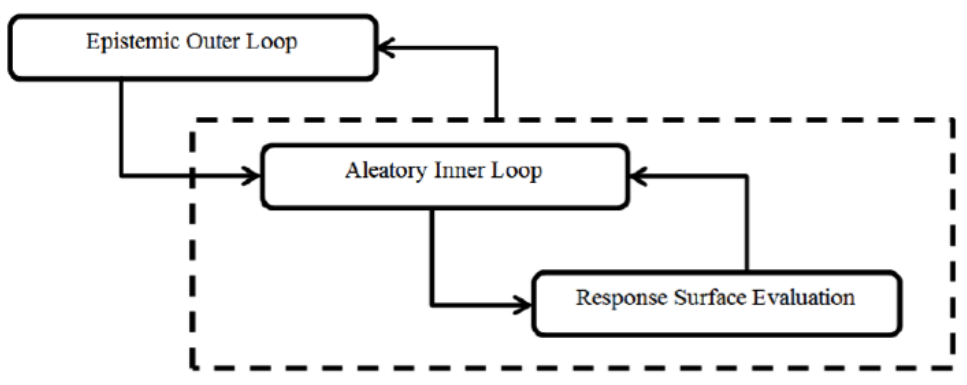

Figure 5: Schematic of Second-Order Probability

As shown by Eldred and Swiler, ${ }^{34}$ the outer (epistemic) loop may also be replaced by optimizationbased interval size determination approaches, which include both local and global optimization methods to determine interval bounds of the output response at selected probability levels. One approach, based on the combination of sampling and local optimization, can be used for the outer loop to determine the bounds at selected probability levels. The outer loop is first evaluated with a small number of samples to determine robust estimates for the initial values of epistemic variables used in local optimization. Then, the optimization is performed with these initial starting points for minimizing or maximizing the response at selected probability levels. This approach provides a computationally efficient means of obtaining accurate results (i.e., interval bounds) via optimization, rather than using extensive sampling of the response surface.

\section{Certification Prediction}

After quantifying the uncertainty in a configuration model, the next step is to determine the reliability of the design under the measured uncertainty. As mentioned in Section I, one of the obstacles of commercial 
supersonic flight is sonic boom production and loudness effect at the ground level. Future regulations on commercial supersonic flight may place limitations on sonic boom loudness measures. One objective of this study is to outline a procedure for predicting certification potential of supersonic configurations.

In order to predict the certification plausibility of a particular configuration a process capability analysis may be employed. ${ }^{35}$ The objective of this analysis is to compare the performance of a process, or in this case the performance metrics (e.g., PLdB, CSEL, etc.), against performance specifications or limits (i.e., certification values). Several factors must be considered, including a margin, measured between a performance metric and a certification value, as well as the uncertainty in a performance metric. For a probabilistic representation of the uncertainty in a particular performance metric, any margin measurement will also carry a probability. Figure 6 illustrates how a margin can be determined from a P-box obtained after the propagation of mixed uncertainty (see Section IV). Notice that the margin is measured between highest response value at a selected probability level or requirement (e.g., 95\% confidence level) and the certification value. A positive margin, shown in Figure 6(a), would exist when the response value (at the probability requirement level) is less than the certification value. On the other hand, if the response value is greater than the certification value, as in Figure 6(c), the margin would be negative and may indicate the design would not pass certification.

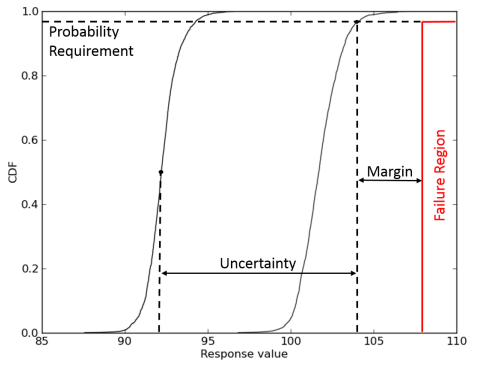

(a) Large Positive Margin

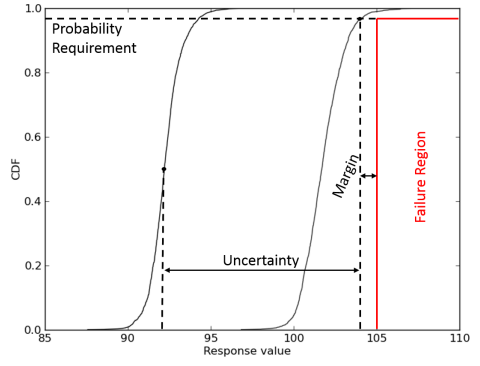

(b) Small Positive Margin

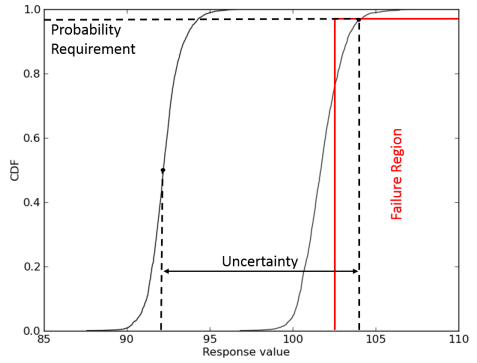

(c) Negative Margin

Figure 6: Certification Predication Margin and Uncertainty Measurements

Because a probability level or requirement is used as part of the margin measurement, it would be possible that the margin may be positive, even if the boundary of the P-box hangs over the "Failure Region" of the certification value, as shown in Figure 6(b). While the margin is positive, the reliability of the configuration performance may be in question. Accounting for this uncertainty is desirable for a reliable design and accurate certification prediction. Using the approach outlined by West et al. ${ }^{36}$ a ratio between the margin and the uncertainty may be used as a reliability measure in the certification prediction. This ratio is termed a confidence ratio, $C R$, and is shown in Eq. (4).

$$
C R=\frac{\text { Margin }}{\text { Uncertainty }}
$$

Note that the measurement of the uncertainty is conservatively taken as the distance between the lower $50 \%$ probability level response value and the upper response value at the probability level of the certification predication analysis. West et al. ${ }^{36}$ also provides details on the treatment of different types of uncertainty representation (i.e., pure aleatory and pure epistemic), as well treatment of cases with uncertainty in the certification values.

This confidence ratio can be viewed as a type of factor of safety. If the margin is larger then the uncertainty in the performance metric, the confidence ratio will be greater than one. In the instance when the uncertainty is greater than the margin, a $C R$ of less than one will result and may indicate weak reliability in the configuration to sufficiently meet a certification requirement. A weak system reliability or failure to meet the certification value (see Figure 6(c)) may warrant changes to the configuration modeling (e.g., reduction of the epistemic uncertainty or a redesign of the actual configuration) or changes in the certification prediction analysis, such as using a lower probability requirement or even reevaluating the certification requirement. 


\section{Uncertainty Quantification and Certification Prediction Framework}

Previous sections of this study describe methodologies of accurate and efficient UQ and certification prediction of low-boom supersonic transport configurations. In order to streamline the analysis, a framework using these methodologies was formulated and is shown in a flowchart in Figure 7. Note that the first step in this framework is a comparison of the CFD model with experimental data. However, in cases where this process is used in tandem with design optimization, experimental data may not exist.

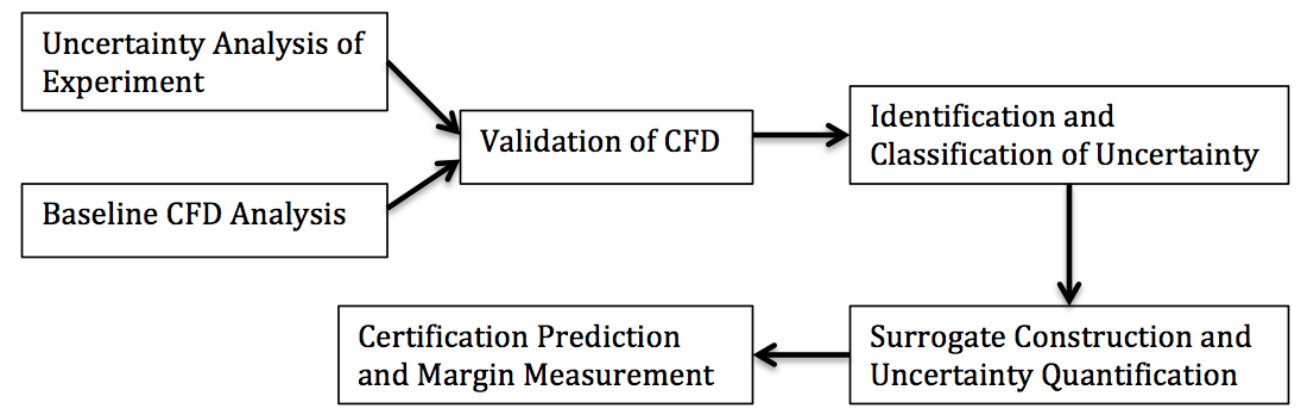

Figure 7: Uncertainty Quantification and Certification Prediction Process

Additionally, because of the use of a surrogate model, checking the accuracy of the surrogate models is necessary. One approach for measuring the accuracy of a surrogate model is to compare response values from the surrogate to a set of sample points distributed throughout the design space, separate from the surrogate model training samples. The objective is to measure the error between response values from the PCE and the actual deterministic model values at the test sample locations. This approach is an indication of the local accuracy of the surrogate model. In this study, sample points from a Latin Hypercube sample structure of the design space and an average of the errors is used as an indication of the surrogate accuracy. Latin Hypercube sampling has the advantage of providing well distributed coverage of the design space with even small sample sizes. Note that each test point used to measure the accuracy of the surrogate model does require an additional evaluation of the deterministic model.

\section{Sources of Uncertainty and Low-Boom Model Analysis Results}

In this section, the UQ and certification prediction framework outlined in the previous section is demonstrated on three low-boom configurations: the SEEB-ALR body of revolution, the NASA $69^{\circ}$ Delta Wing heritage model, and the Lockheed Martin 1021-01 configuration. The section begins with the identification of possible sources of uncertainty in the modeling approach and in low-boom configuration operation (i.e., in flight uncertainty). Then, the framework is demonstrated on each of the configurations with detailed discussion of output uncertainty in design parameters of interest. Note that for each of the configurations, the experimental data used to validate the CFD models was taken from wind tunnel test data discussed in detail by Cliff et al. ${ }^{37}$

Additionally, a sensitivity analysis was performed on each configuration to identify the uncertain parameters that contribute most significantly to the total output variance. This information can be used for multiple purposes including targeting resources for improvement of models, refinement of uncertain information, or in design. The parameter sensitivities were determined using a global nonlinear approach called Sobol indices and is based on the PCE. Further details regarding this approach are given by Sudret, ${ }^{38}$ Crestaux et al., ${ }^{39}$ as well as Ghaffari et al. ${ }^{40}$

\section{A. Sources of Uncertainty in Low-Boom Configuration Modeling}

One of the most critical steps in any uncertainty analysis is the identification and classification of the input uncertainties of the models. In this study, the deterministic model is decomposed into two parts as outlined in section II. First, for the CFD model, two input uncertain parameters were identified as possible sources of uncertainty: Mach number and angle of attack. The type and amount of uncertainty are shown in Table 2. Note that these parameters were determined to be aleatory parameters given their possible inherent nature 
as small fluctuations in these parameters are typically unavoidable. For the Mach number, two mean values are considered for comparison with the experimental data. The SEEB-ALR and LM 1021-01 configurations were tested at Mach 1.6, while the Delta Wing was tested at Mach 1.7.

Table 2: CFD Aleatory Input Parameters

\begin{tabular}{cccc}
\hline \hline Input & Distribution & Mean & Std. Dev. \\
\hline Angle of Attack & Gaussian & 0.0 & 0.1 \\
Mach Number & Gaussian & $1.6 / 1.7$ & 0.0016 \\
\hline \hline
\end{tabular}

A second set of uncertain parameters were identified as coming from the propagation model, sBOOM. ${ }^{20}$ This model contained a particularly large amount of uncertainty, both aleatory and epistemic, due to its complexity and the large number of tunable input parameters. The aleatory parameters, listed in Table 3 consist of two groups. The first is uncertainty in the atmosphere that may effect the propagation of the sonic boom signature as it travels from the vehicle near-field to the ground. The second group of parameters are those that may effect the shape of the signature due to changes in vehicle orientation and orientation rates. The measurement location or azimuth is also considered uncertain. In this study, only the on-track position, with uncertainty, is investigated.

Table 3: sBOOM Aleatory Input Parameters

\begin{tabular}{cccc}
\hline \hline Input & Distribution & Mean & Std. Dev. \\
\hline Temperature Profile (\%) & Gaussian & 1.0 & 0.01 \\
Humidity Profile (\%) & Gaussian & 1.0 & 0.01 \\
Climb Angle (Deg.) & Gaussian & 0.0 & 0.1 \\
Azimuth (Deg.) & Gaussian & 0.0 & 0.1 \\
Turn Rate (Deg./s) & Gaussian & 0.0 & 0.05 \\
Climb Rate (Deg./s) & Gaussian & 0.0 & 0.05 \\
\hline \hline
\end{tabular}

As with the CFD uncertain parameters, the aleatory parameters are those that have been modeled, but random fluctuations may still occur. However, epistemic parameters, listed in Table 4, exist due to lack of knowledge of the correct modeling approach. The initial step size and signature propagation points parameters are adjustable sBOOM specific input parameters. The signature propagation points variable is related to the sampling frequency of the signal and may directly effect the accuracy of the loudness metrics from the integrated ground level signature. The number of points necessary for an accurate result may vary based on the signal length, signal shape, or the signal source (i.e., the configuration). Note also that the value of this parameter is both the number of points used within the propagation routine and the number of points in the final ground level signature that is analyzed to find the loudness measures. The other two parameters (reflection factor and ground level altitude) are considered epistemic uncertain parameters as they are not being modeled for a specific flight location. These parameters may vary significantly along a flight path.

Table 4: sBOOM Epistemic Input Parameters

\begin{tabular}{ccc}
\hline \hline Input & Min. & Max. \\
\hline Initial Step Size & 0.007 & 0.03 \\
Reflection Factor & 1.8 & 2.0 \\
Ground Elevation (ft) & 0.0 & 5000.0 \\
Signature Propagation Points & 20000 & 60000 \\
\hline \hline
\end{tabular}


In addition to the above uncertain input parameters, inviscid and fully turbulent CFD solutions were performed for all of the configurations analyzed in this study. The purpose of this is to quantify the effect of different modeling fidelities for the various low-boom configurations studied. Note that the amount of uncertainty in each parameter discussed above was the result of much discussion among the authors and other experts in the field.

\section{B. SEEB-ALR Body of Revolution}

\section{Comparison with Experiment}

The first step in the UQ process is to validate the CFD solution. Near-field signatures were taken at 21.2 inches from the body to coincide with experimental data. Comparison with the experiment is shown in Figure 8(a) for both as-built and as-designed geometries, and for both inviscid and fully turbulent flow assumptions. A residual scale plot of the signatures is shown in Figure 8(b). In this figure, the averaged experimental signature is treated as a reference and is subtracted off of the uncertainty bounds and the CFD signatures. Notice that there is good agreement between the experiments and the CFD results as the CFD signatures mostly lie within the bounds of the uncertainty in the experimental results. There are some differences in the peaks of the signatures, but the experimental results have rounded peaks due to the measurement approach. ${ }^{37}$ This agreement is deemed to be acceptable and is assumed to validate the baseline numerical solution.

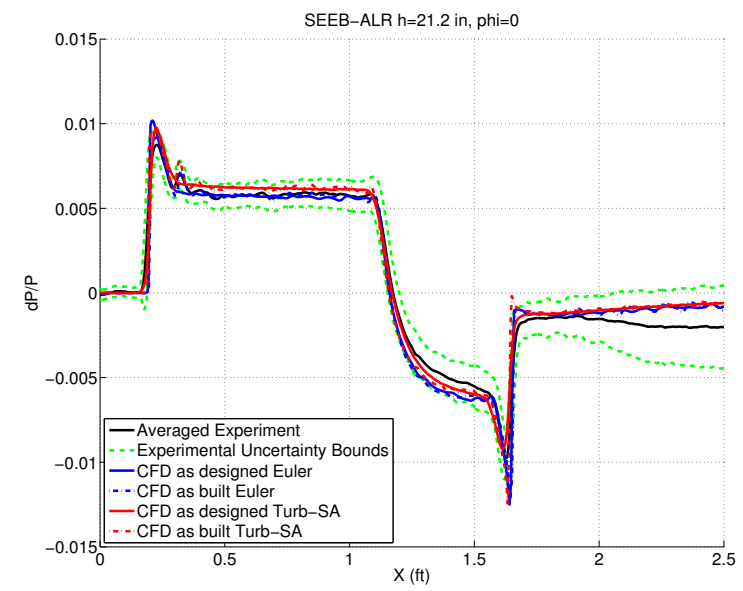

(a) Signatures

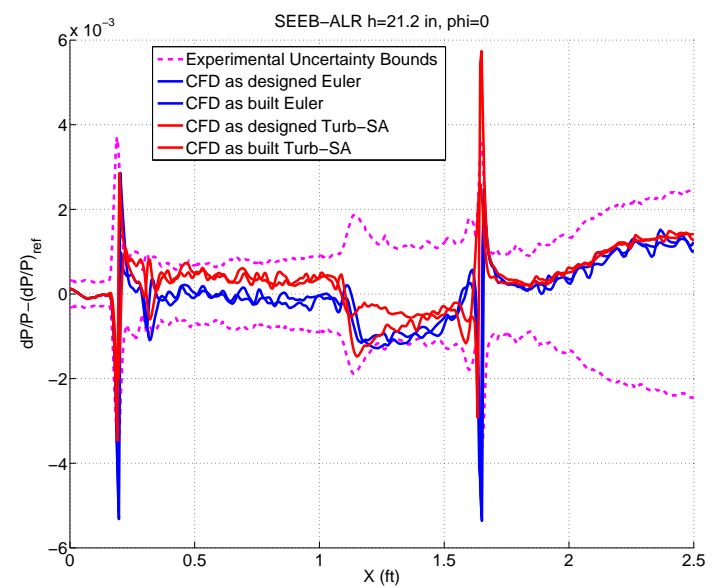

(b) Residual Scale

Figure 8: SEEB-ALR Near-Field CFD Comparison with Experimental Results

\section{Uncertainty Quantification}

After validating the CFD model, the next step is to construct the surrogate model(s) that represent the design quantities of interest. In this study, PLdB and CSEL were considered. In total, there are 12 uncertain parameters, as discussed above. Using Eq. (2), 182 evaluations of the deterministic model were necessary to construct a second order PCE with twice oversampling. In evaluating the deterministic model, the first step is to obtain CFD solutions for the near-field pressure signatures. The dispersion of the deterministic samples for the Euler as-built case are shown in Figure 9(a). Figure 9(b) shows the same dispersion of signals, but shifted to reference location for better visualization of changes in signature amplitude.

The CFD signatures were then each propagated through SBOOM with the additional uncertainty that was identified for this model. For the Euler as-built case, the resulting ground signature dispersion is shown in Figure 10(a) and shifted to a reference location in Figure 10(b). The ground signatures were then analyzed to produce the desired loudness quantities of interest.

Similar dispersions of the near-field and ground level signatures can be generated for the other three cases investigated for this model (Euler as-designed, turbulent as-built and turbulent as-designed). While not shown here, these dispersions are similar to those shown for the Euler as-built case. 


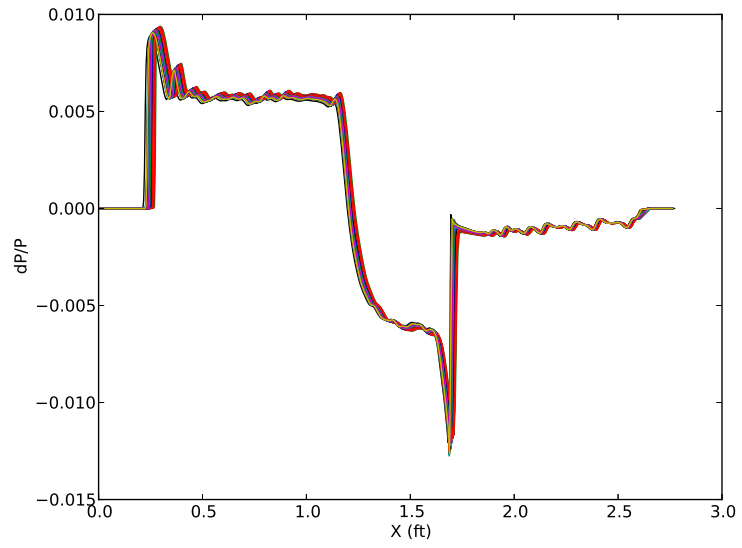

(a) Actual

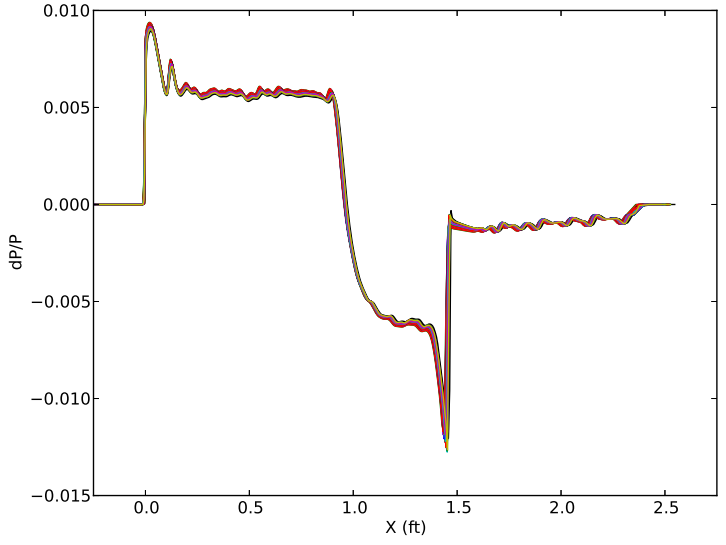

(b) Shifted

Figure 9: SEEB-ALR Euler as-Built Near-Field Pressure Signature Dispersion

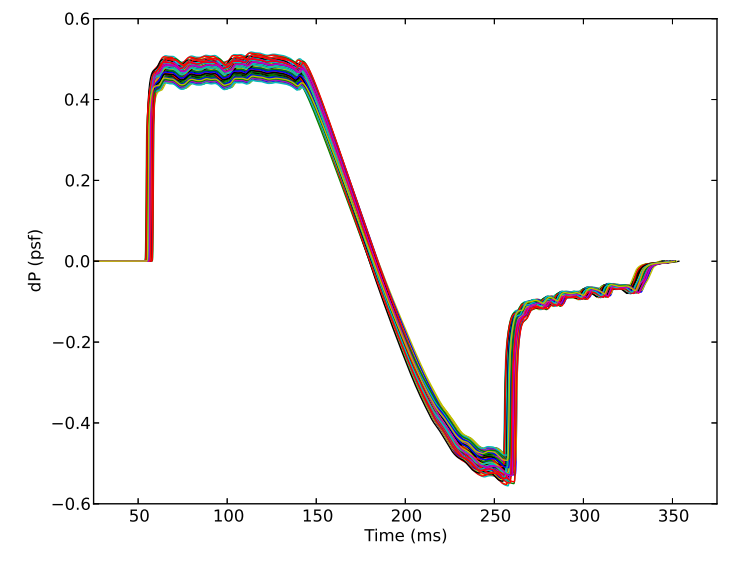

(a) Actual

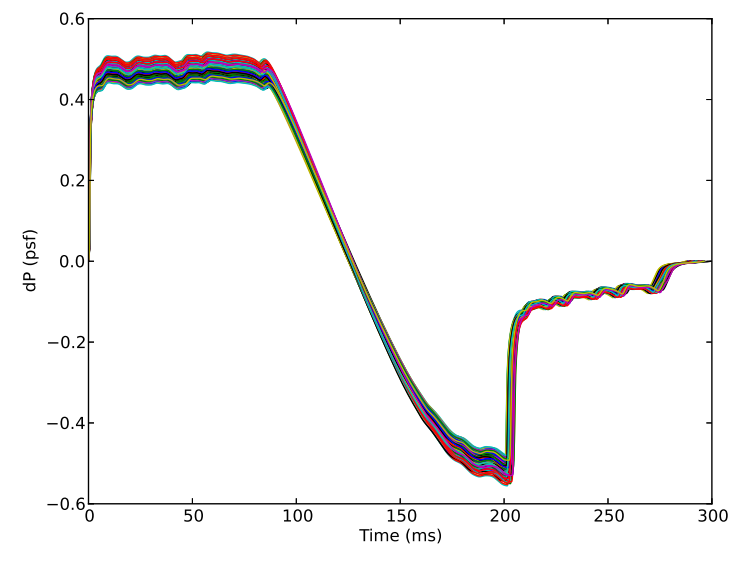

(b) Shifted

Figure 10: SEEB-ALR Euler as-Built Ground Signature Dispersion

Note that for most of the CFD models, the signatures do not recover fully. This can stem from the inclusion of the mounting system used within the wind tunnel in the computational geometry and/or the truncation of the computational domain. For more accurate results and to eliminate any numerical instabilities within the propagation routine/loudness measure calculations, the ends of the signatures are forced to zero and additional zero padding is added to the signatures for all of the configurations in this study.

At this point, the surrogate models for the output quantities of interest can be created by solving the linear system of equations given in Eq. (3). Measuring the accuracy of the surrogate models across the design space can be done by sampling a set of test points in the design space and comparing outputs from the deterministic model with the outputs from the surrogates at the same sample locations. Twenty new samples were taken to measure the accuracy of the surrogates. It was determined that the average error at these twenty test points was less than $0.03 \%$ for all of the geometry/flow type/loudness quantity combinations indicating the selection of a second-order PCE was sufficient. Note that these test points are different from those points used to train the surrogate models and are distributed evenly throughout the design space with a Latin Hypercube sample structure.

With the surrogate models constructed and validated, the uncertainty can be propagated through the surrogates using the second-order probability analysis outlined in section IV. This was done using the sampling approach, resulting in the family of CDFs. For the Euler as-built case, the boundaries of the 
P-boxes are shown in Figure 11(a) and Figure 11(b) for PLdB and CSEL, respectively.

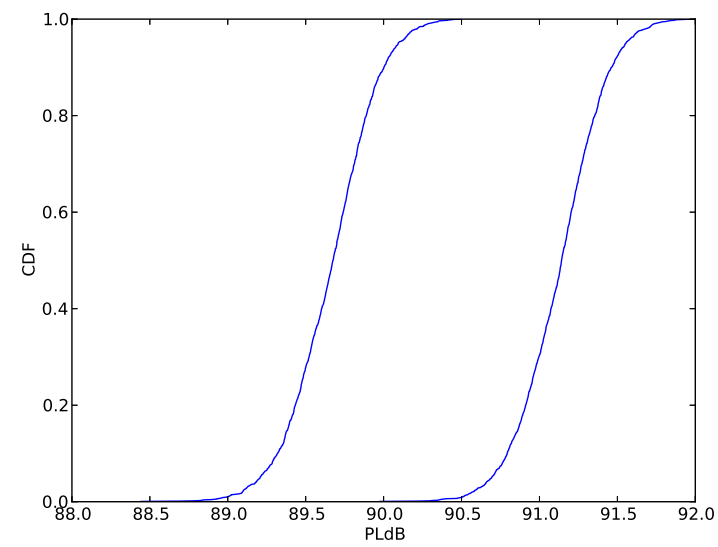

(a) PLdB

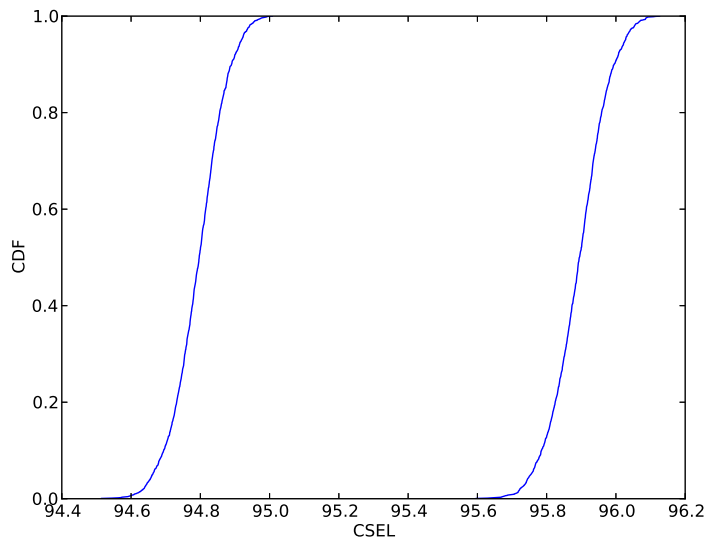

(b) CSEL

Figure 11: SEEB-ALR Euler as-Built PLdB and CSEL Probability Box Boundaries

A significant amount of information can be disseminated from P-boxes, including confidence intervals. For the case of mixed uncertainty, one approach to obtaining the $95 \%$ confidence interval, for example, is to take the upper $97.5 \%$ probability level and the lower $2.5 \%$ probability level as the interval. A summary of the $95 \%$ confidence intervals is shown in Table 5 for all of the models, subject to the uncertainty identified in this study.

Table 5: SEEB-ALR 95\% Confidence Intervals

\begin{tabular}{ccc}
\hline \hline Configuration & PLdB & CSEL \\
\hline Euler as-Built & {$[89.12,91.63]$} & {$[94.64,96.05]$} \\
Euler as-Designed & {$[88.06,90.49]$} & {$[94.32,95.80]$} \\
Turbulent as-Built & {$[89.44,91.95]$} & {$[94.78,96.22]$} \\
Turbulent as-Designed & {$[88.98,91.61]$} & {$[94.75,96.20]$} \\
\hline \hline
\end{tabular}

From these results, it can been seen that there is a difference between the ideal as-designed geometry and the actual as-built model. The imperfections in the surface cause an increase in the PLdB level. In the case of CSEL, the same trend is not as severe, but still present. Additionally, it can be disseminated that the effect of an inviscid versus the fully turbulent analysis is small and is less than that of the effect of the surface imperfections in the as-built geometry. For preliminary design and analysis, this indicates the use of the low fidelity solution may be acceptable when considering the added computational cost of a fully turbulent solution, for this configuration.

\section{Certification Prediction}

The final step in the analysis is to predict whether or not the design, under uncertainty, could potentially pass a certification based on the design quantity of interest. Currently, the actual certification value for low-boom configurations is unknown. However, the methodology outlined in section $\mathrm{V}$ can be easily implemented for certification prediction. For example, if the certification requirement was a PLdB of less than 100, the Euler as-built design would have a CR of 4.27 for a $95 \%$ confidence analysis. This may indicate that the margin is sufficiently larger than the uncertainty in the design. 


\section{Sensitivity Analysis}

A sensitivity analysis of the uncertainty parameters was performed to highlight which of the parameters contribute most significantly to the total uncertainty. The contribution of the top uncertain parameters to both PLdB and CSEL are given in Tables 6 and 7, respectively. For both metrics, the reflection factor appears to be the most dominate of the parameters considered. This is especially true for CSEL as the reflection factor contributes about 85 to $90 \%$ to the total output variance. For PLdB, a significant amount of uncertainty also arises due to the uncertainty in the atmospheric humidity profile. Loubeau and Coulouvrat ${ }^{41}$ have shown that variation in the atmospheric humidity can significantly effect the sonic-boom rise time, which may effect the signature loudness.

Table 6: SEEB-ALR Top Uncertain Parameter Contribution to PLdB Total Uncertainty

\begin{tabular}{ccccc}
\hline \hline Uncertain Parameter & $\begin{array}{c}\text { Euler } \\
\text { as-Built }\end{array}$ & $\begin{array}{c}\text { Euler } \\
\text { as-Designed }\end{array}$ & $\begin{array}{c}\text { Turbulent } \\
\text { as-Built }\end{array}$ & $\begin{array}{c}\text { Turbulent } \\
\text { as-Designed }\end{array}$ \\
\hline Angle of Attack & $4.7 \%$ & $9.6 \%$ & $2.4 \%$ & $6.7 \%$ \\
Initial Step Size & $1.6 \%$ & $1.1 \%$ & $1.7 \%$ & $1.8 \%$ \\
Reflection Factor & $46.4 \%$ & $44.8 \%$ & $45.9 \%$ & $44.2 \%$ \\
Humidity Profile & $38.3 \%$ & $35.7 \%$ & $41.6 \%$ & $36.1 \%$ \\
Ground Elevation & $7.9 \%$ & $7.7 \%$ & $6.8 \%$ & $9.7 \%$ \\
All Others & $<1 \%$ & $<1 \%$ & $<1 \%$ & $<1 \%$ \\
\hline \hline
\end{tabular}

Table 7: SEEB-ALR Top Uncertain Parameter Contribution to CSEL Total Uncertainty

\begin{tabular}{ccccc}
\hline \hline Uncertain Parameter & $\begin{array}{c}\text { Euler } \\
\text { as-Built }\end{array}$ & $\begin{array}{c}\text { Euler } \\
\text { as-Designed }\end{array}$ & $\begin{array}{c}\text { Turbulent } \\
\text { as-Built }\end{array}$ & $\begin{array}{c}\text { Turbulent } \\
\text { as-Designed }\end{array}$ \\
\hline Angle of Attack & $3.6 \%$ & $6.2 \%$ & $4.5 \%$ & $4.6 \%$ \\
Reflection Factor & $88.2 \%$ & $84.1 \%$ & $86.5 \%$ & $86.0 \%$ \\
Temperature Profile & $2.2 \%$ & $2.4 \%$ & $2.4 \%$ & $2.4 \%$ \\
Humidity Profile & $1.7 \%$ & $1.5 \%$ & $1.7 \%$ & $1.7 \%$ \\
Ground Elevation & $4.1 \%$ & $5.5 \%$ & $4.6 \%$ & $5.2 \%$ \\
All Others & $<1 \%$ & $<1 \%$ & $<1 \%$ & $<1 \%$ \\
\hline \hline
\end{tabular}

\section{NASA $69^{\circ}$ Delta Wing}

\section{Comparison with Experiment}

For the NASA $69^{\circ}$ Delta Wing, near-field signatures were taken at 24.8 inches from the body to coincide with experimental data. Comparison with the experiment is shown in Figure 12(a) for both inviscid and fully turbulent flow assumptions. A residual scale plot of the signatures is shown in Figure 12(b). The CFD signatures are in fairly good agreement with the experimental results as the signatures lie primarily within the bounds of the uncertainty in the experimental results. There is some rounding of the experimental results similar to the results of the SEEB-ALR model. This is due to model vibration and the instrumentation used during the wind tunnel testing. ${ }^{37}$ However, given these small and expected differences, the baseline numerical solution is considered to be accurate and validated for the purposes of this study.

\section{Uncertainty Quantification}

As above, surrogate models are constructed for the design quantities of interest, PLdB and CSEL. The number of uncertain parameters is the same as with the SEEB-ALR and, therefore, the deterministic model 


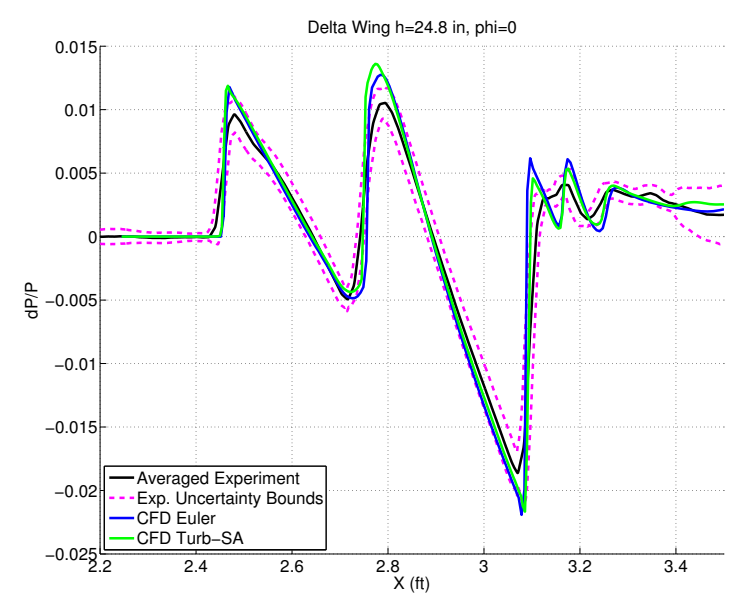

(a) Signatures

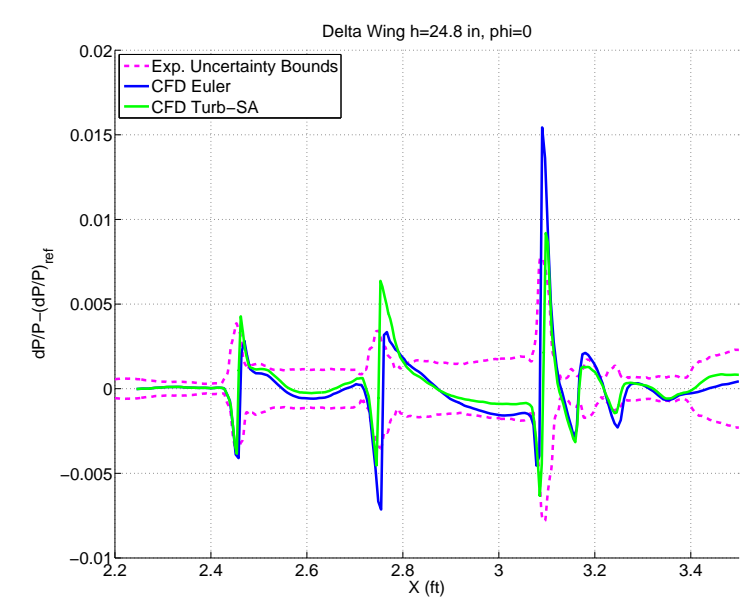

(b) Residual Scale

Figure 12: $69^{\circ}$ Delta Wing Near-Field CFD Comparison with Experimental Results

is evaluated 182 times to construct a second order PCE. The dispersion of the deterministic samples for the Euler case are shown in Figure 13(a). Figure 13(b) shows the same dispersion of signals, but shifted to reference location for better visualization of changes in signature amplitude. The resulting ground signature dispersion, for the Euler case, is shown in Figure 14(a) and shifted to a reference location in Figure 14(b). Similar dispersions of the near-field and ground level signatures can be generated for the fully turbulent case. While not shown here, this dispersions are similar to those shown above for the Euler case.

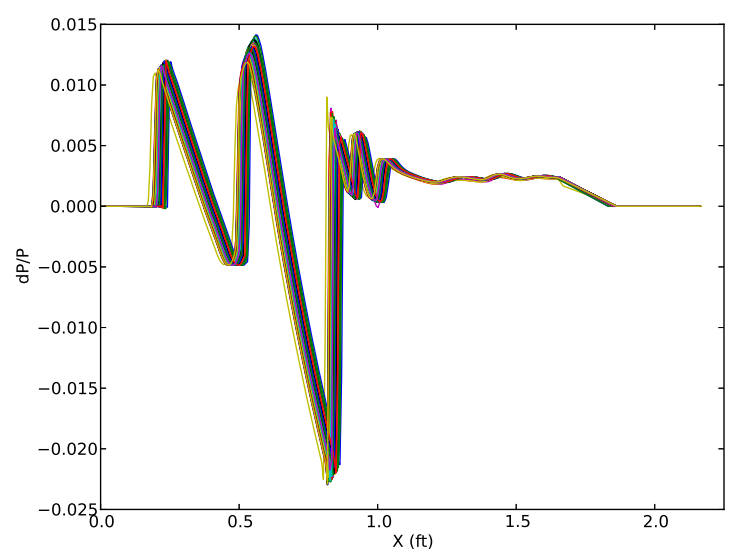

(a) Actual

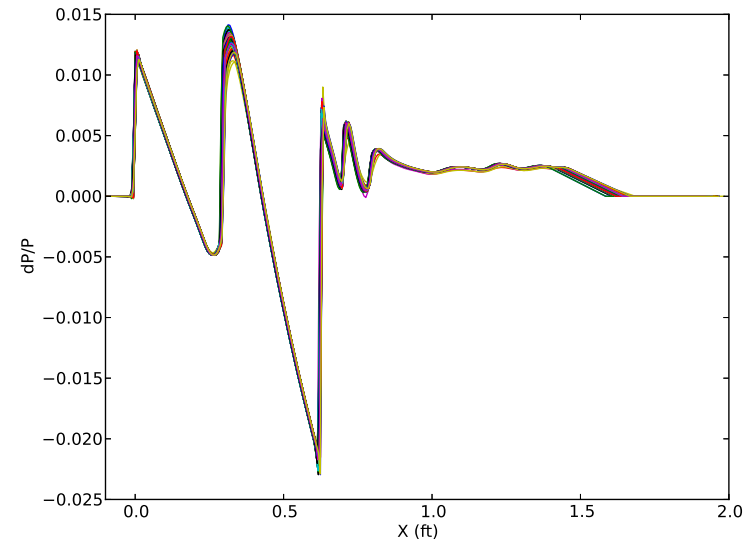

(b) Shifted

Figure 13: $69^{\circ}$ Delta Wing Euler Near-Field Pressure Signature Dispersion

As with the SEEB-ALR, the accuracy of the surrogate model needs to be verified. It was determined that the average error at the 20 test points was less than $0.4 \%$ for all of the flow type/loudness quantity combinations indicating the selection of a second-order PCE was sufficient. With the surrogate models constructed and validated, the uncertainty can be propagated through the surrogates using the second-order probability analysis outlined in section IV. This was done using the sampling approach, resulting in the family of CDFs. For the Euler case, the boundaries of the probability boxes are shown in Figure 15(a) and Figure 15(b) for PLdB and CSEL, respectively.

A summary of the $95 \%$ confidence intervals for both Euler and Turbulent cases is shown in Table 8. From these results, it can be seen that the effect of an inviscid versus the fully turbulent analysis is slightly more significant compared to the SEEB-ALR model for both loudness measures. However, there is still less than 


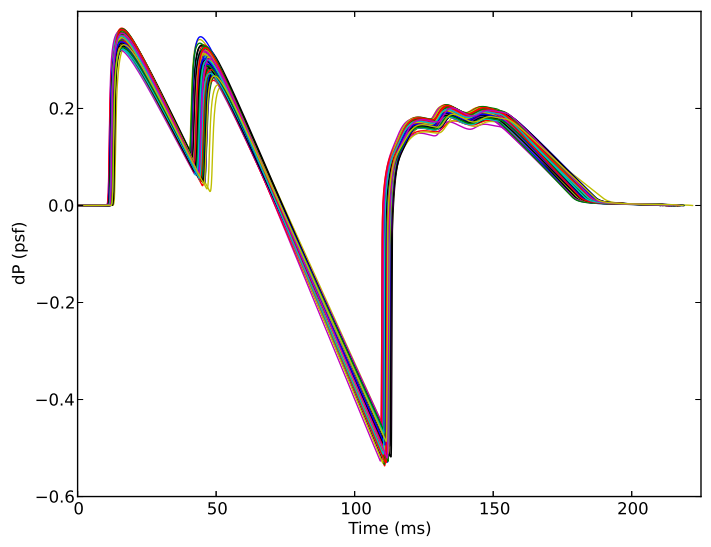

(a) Actual

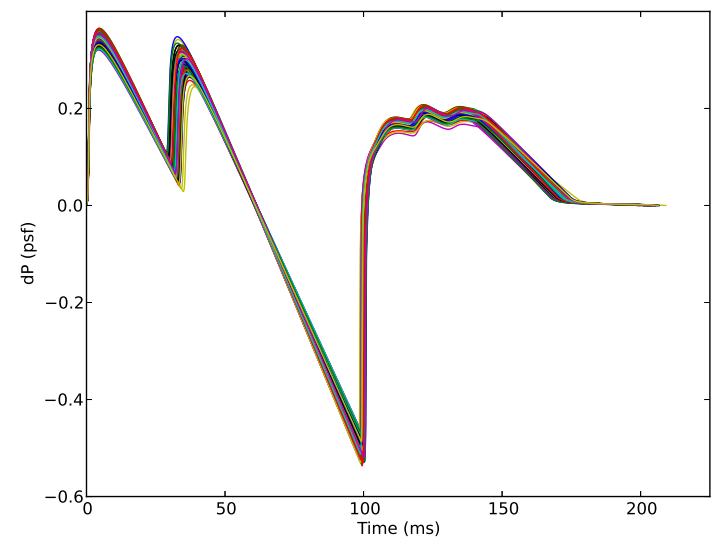

(b) Shifted

Figure 14: $69^{\circ}$ Delta Wing Euler Ground Signature Dispersion

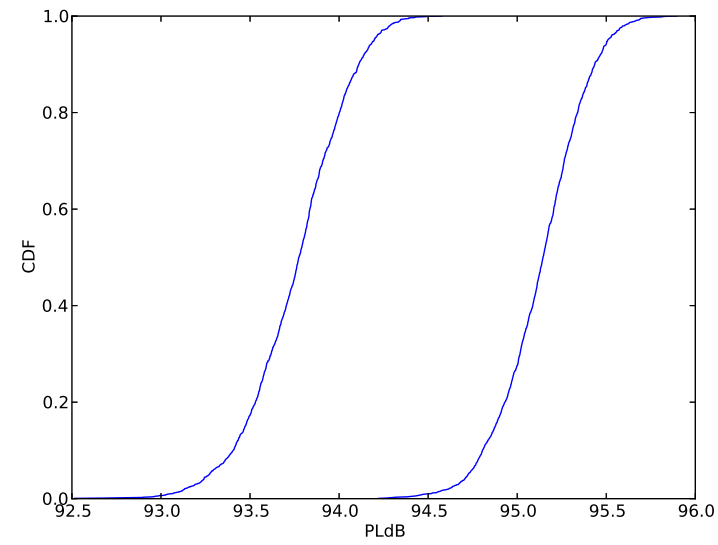

(a) PLdB

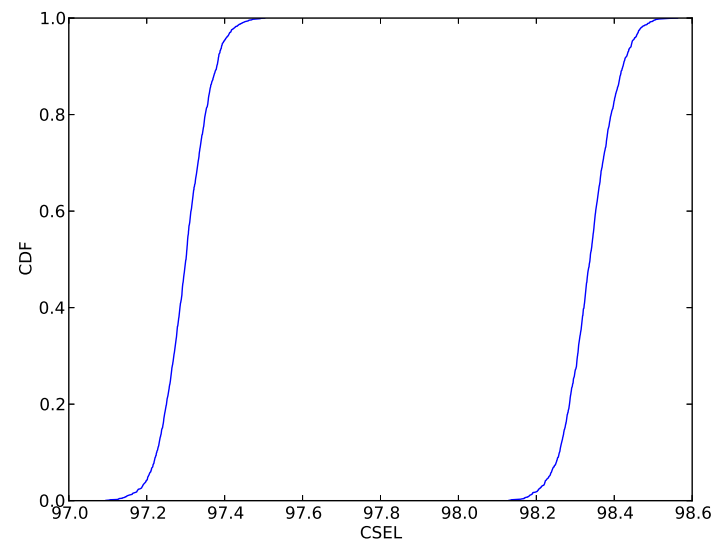

(b) CSEL

Figure 15: $69^{\circ}$ Delta Wing Euler PLdB and CSEL Probability Box Boundaries

a one PLdB and CSEL difference between the two levels of model fidelity. This may indicate that the use of a Euler analysis may be sufficient given the reduce computational cost over the fully turbulent analysis, for this model.

Table 8: $69^{\circ}$ Delta Wing 95\% Confidence Intervals

\begin{tabular}{ccc}
\hline \hline Configuration & PLdB & CSEL \\
\hline Euler & {$[93.16,95.58]$} & {$[97.18,98.46]$} \\
Turbulent & {$[94.03,96.35]$} & {$[97.63,98.85]$} \\
\hline \hline
\end{tabular}

\section{Sensitivity Analysis}

The contribution of the top uncertain parameters to both PLdB and CSEL are given in Tables 9 and 10, respectively. The results of this sensitivity analysis are similar to those observed for the SEEB-ALR. The reflection factor contributes even more to the total output uncertainty for both loudness metrics, and the 
contribution of the atmospheric humidity variation contributes significantly to PLdB.

Table 9: $69^{\circ}$ Delta Wing Top Uncertain PaTable 10: $69^{\circ}$ Delta Wing Top Uncertain Parameter Contribution to PLdB Total Uncertainty rameter Contribution to CSEL Total Uncertainty

\begin{tabular}{ccc}
\hline \hline Uncertain Parameter & Euler & Turbulent \\
\hline Initial Step Size & $1.4 \%$ & $1.0 \%$ \\
Reflection Factor & $50.9 \%$ & $52.0 \%$ \\
Temperature Profile & $1.3 \%$ & $1.8 \%$ \\
Humidity Profile & $37.1 \%$ & $38.0 \%$ \\
Ground Elevation & $7.9 \%$ & $6.3 \%$ \\
All Others & $<1 \%$ & $<1 \%$ \\
\hline \hline
\end{tabular}

\begin{tabular}{ccc}
\hline \hline Uncertain Parameter & Euler & Turbulent \\
\hline Reflection Factor & $93.1 \%$ & $94.4 \%$ \\
Temperature Profile & $2.1 \%$ & $2.5 \%$ \\
Humidity Profile & $1.1 \%$ & $1.5 \%$ \\
Ground Elevation & $1.9 \%$ & $1.4 \%$ \\
All Others & $<1 \%$ & $<1 \%$ \\
\hline \hline
\end{tabular}

\section{LM 1021-01 Low-Boom Configuration}

\section{Comparison with Experiment}

For the LM 1021-01, near-field signatures were taken at 20.7 inches from the body to coincide with experimental data. Comparison with the experiment is shown in Figure 16(a) for both inviscid and fully turbulent flow assumptions. A residual scale plot of the signatures is shown in Figure 16(b). The fully turbulent solution agrees well with the experimental results, with the exception of the rounding of the peaks in the experimental results, similar to the delta wing and SEEB-ALR models. The inviscid solution, however, does not agree as well. Aftosmis et al. ${ }^{18}$ identify the source of this discrepancy, which stems from a shock originating at the front of the under wing nacelle. In the viscous case, this shock is smeared by the boundary layer between the wing and nacelle, but propagates in the inviscid simulation. A more complete discussion is included in the reference. An important note should be made regarding the length of the LM 1021-01 signature. The computational domain is slightly truncated as the signature does not fully recover to zero. As stated above, the ends of the signatures are forced to zero to prevent any numerical issues in propagating the signature to the ground level. This artificial forcing of the signature, however, is not expected to effect the loudness results as this is not in a strong shock or expansion region.

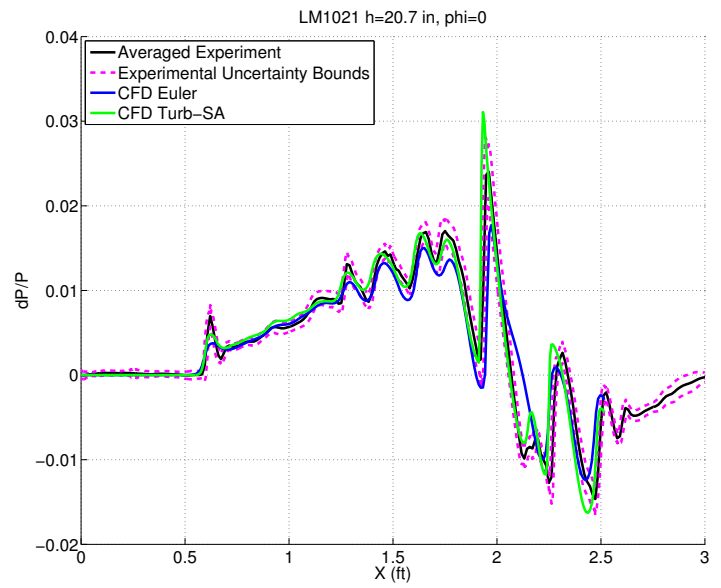

(a) Signatures

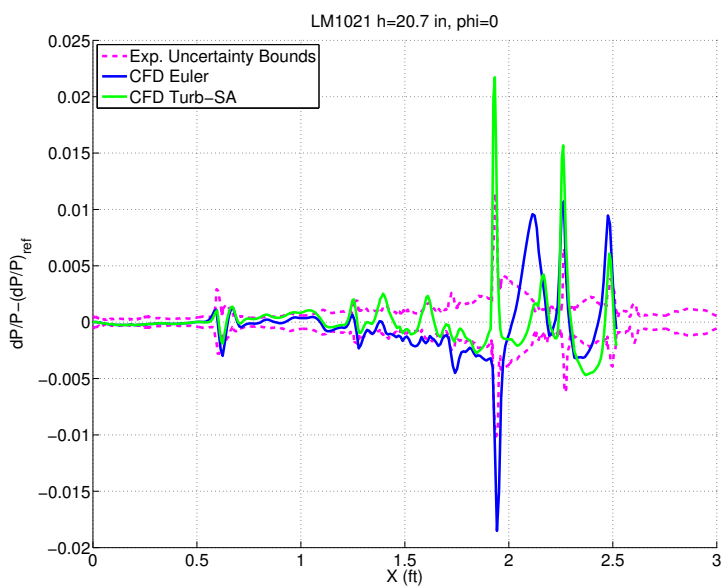

(b) Residual Scale

Figure 16: LM 1021-01 Near-Field CFD Comparison with Experimental Results 


\section{Uncertainty Quantification}

The surrogate models for CSEL and PLdB are constructed in the same fashion as for the other cases. The dispersion of the deterministic samples for the Euler case are shown in Figure 17(a). Figure 17(b) shows the same dispersion of signals, but shifted to reference location for better visualization of changes in signature amplitude. For the Euler case, the resulting ground signature dispersion is shown in Figure 18(a) and shifted to a reference location in Figure 18(b). The ground signatures were then analyzed to produce the desired loudness quantities of interest.

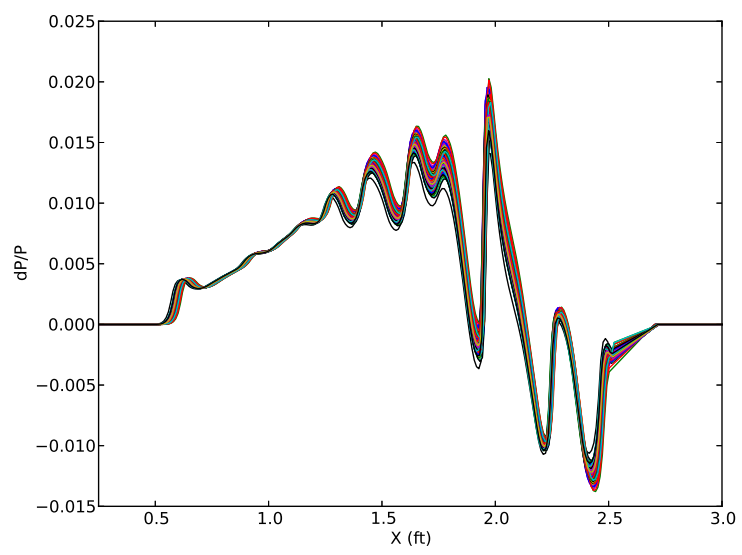

(a) Actual

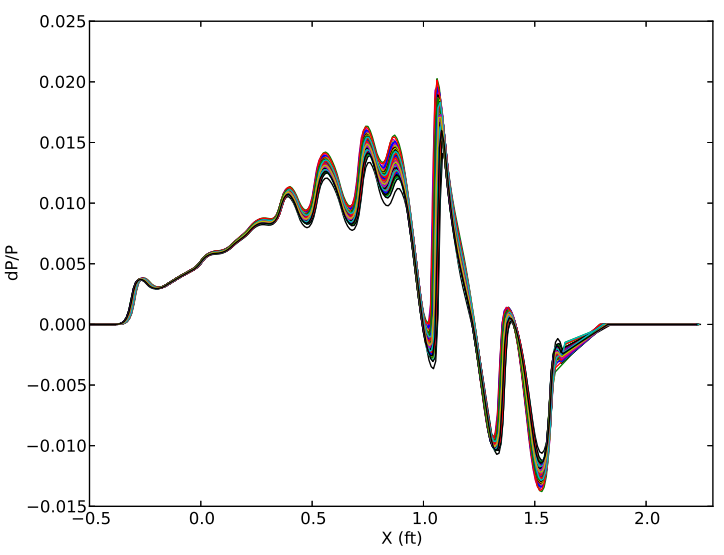

(b) Shifted

Figure 17: LM 1021-01 Euler Near-Field Pressure Signature Dispersion

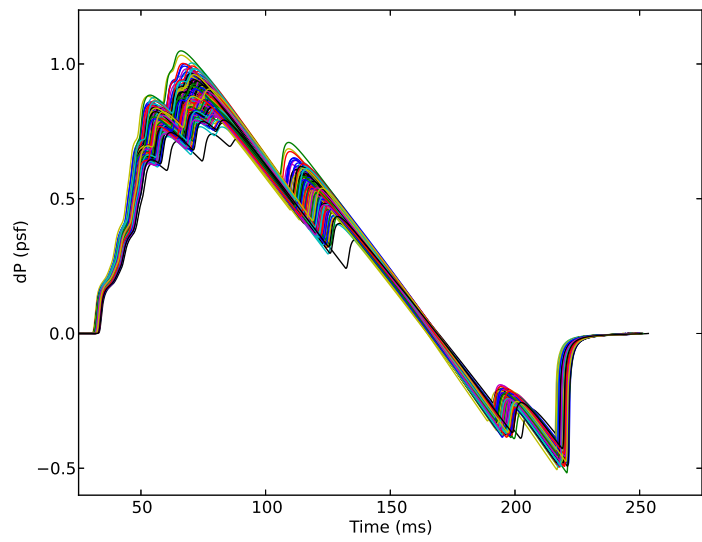

(a) Actual

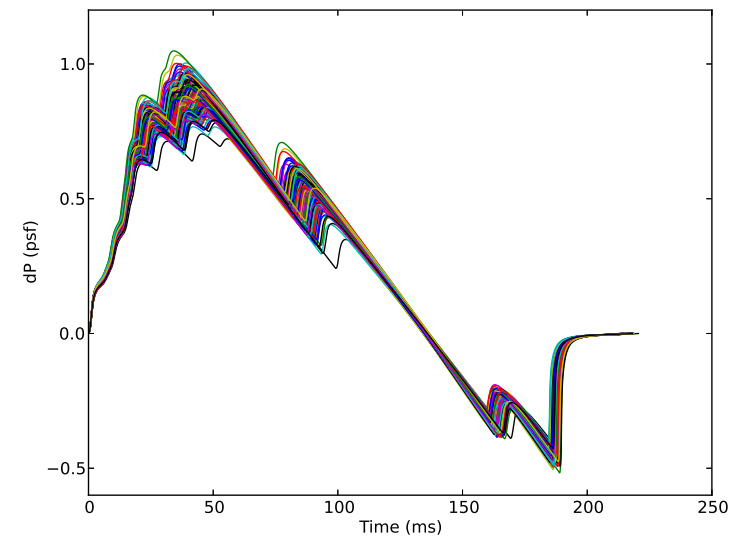

(b) Shifted

Figure 18: LM 1021-01 Euler Ground Signature Dispersion

Similar dispersions of the near-field and ground level signatures can be generated for the fully turbulent case. Because of the effect of different flow physics modeling, the signatures for the turbulent case are slightly different, both in the near-field, as well as at the ground level. The near-field signatures are shown in Figure 19(a) and shifted to a reference location in Figure 19(b). The ground level signatures are shown in Figure 20(a) and shifted to a reference location in Figure 20(b).

The surrogates are validated using 20 test points distributed throughout the design space, similar to the previous two models. The average error at these 20 test points was less than $0.2 \%$ for all of the flow type/loudness quantity combinations indicating the selection of a second-order PCE was sufficient. The uncertainty is propagated through the surrogates and a family of CDFs were produced. For the Euler case, 


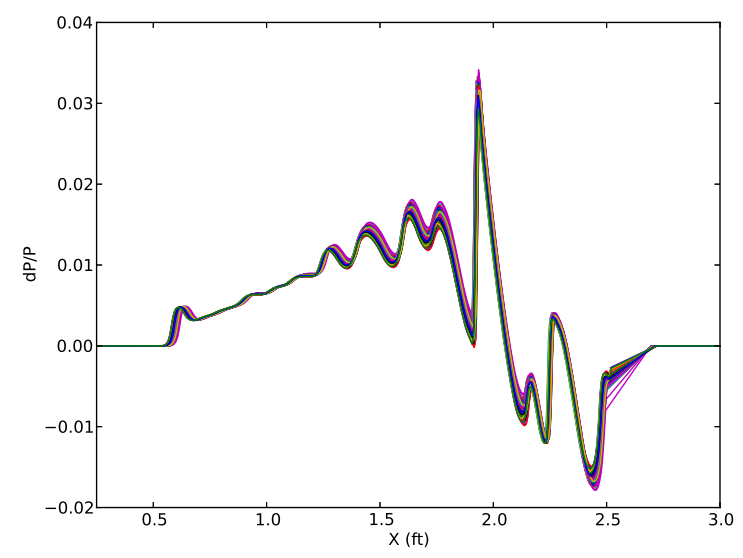

(a) Actual

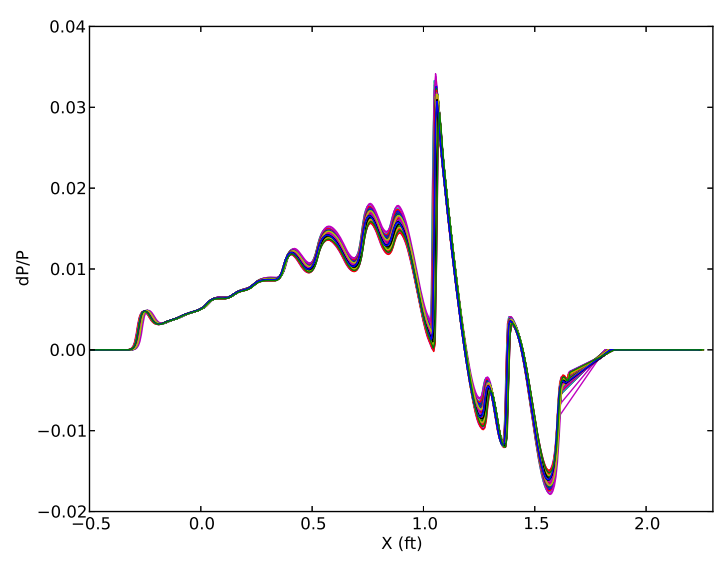

(b) Shifted

Figure 19: LM 1021-01 Turbulent Near-Field Pressure Signature Dispersion

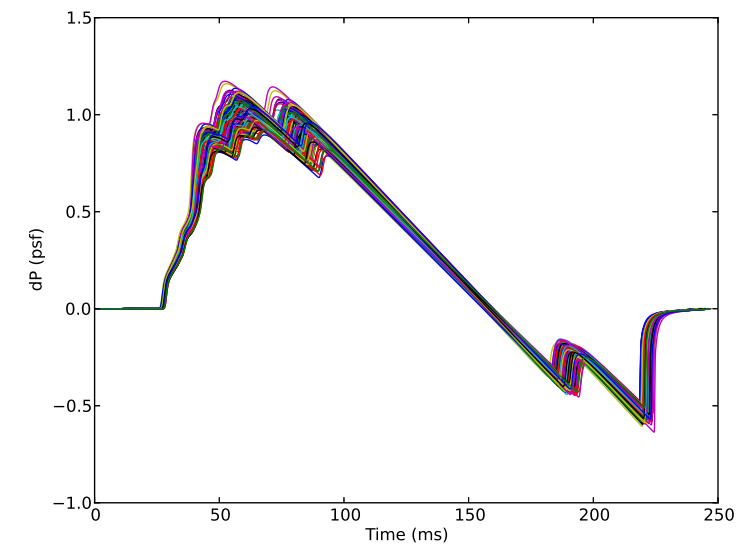

(a) Actual

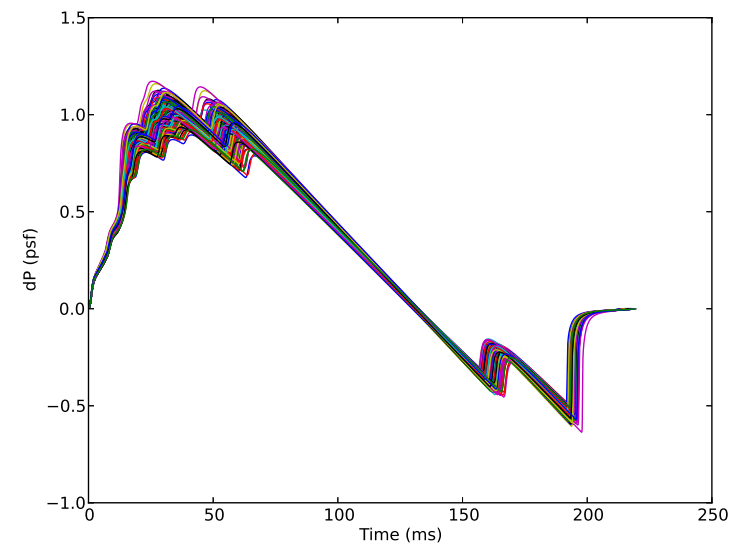

(b) Shifted

Figure 20: LM 1021-01 Turbulent Ground Signature Dispersion

the boundaries of the probability boxes are shown in Figure 21(a) and Figure 21(b) for PLdB and CSEL, respectively.

A summary of the $95 \%$ confidence intervals for both Euler and Turbulent cases is shown in Table 11. From these results, it can be seen that the effect of an inviscid versus the fully turbulent analysis is more significant than for the SEEB-ALR and the Delta wing models for both loudness measures. This is due to the reduced order modeling fidelity of the Euler analysis. While the loudness values are lower for the inviscid case, which is desirable, the accuracy of the Euler model has to be in question due to this significant difference. Along with the discrepancy in the near-field signature, this indicates an inviscid assumption is not appropriate for this configuration under the current flow conditions. Aftosmis et al. ${ }^{18}$ notes the wind tunnel experiments were conducted at a relatively low Reynolds number (which was matched for the simulations). At a higher Reynolds number, an Euler solution may be sufficient, but this must first be validated for the new freestream conditions.

\section{Sensitivity Analysis}

The contribution of the top uncertain parameters to both PLdB and CSEL are given in Tables 12 and 13, respectively. The results of this sensitivity analysis is different than that observed for the SEEB-ALR 


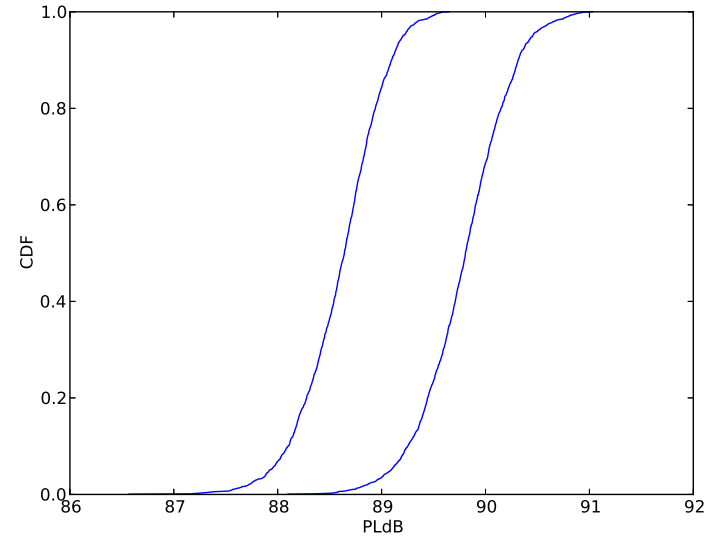

(a) PLdB

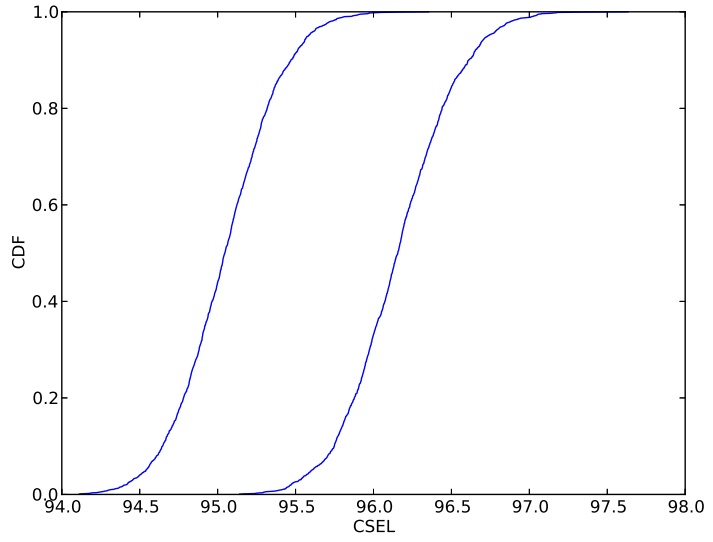

(b) CSEL

Figure 21: LM 1021-01 Euler PLdB Probability Box Boundaries

Table 11: LM 1021-01 95\% Confidence Intervals

\begin{tabular}{ccc}
\hline \hline Configuration & PLdB & CSEL \\
\hline Euler & {$[87.76,90.60]$} & {$[94.43,96.85]$} \\
Turbulent & {$[90.17,93.79]$} & {$[96.06,98.76]$} \\
\hline \hline
\end{tabular}

and Delta Wing models as the reflection factor is no long the top parameter. While the reflection is still significant, angle of attack now dominates the output uncertainty for both loudness metrics. Because of the specific design of the LM 1021-01, changes in angle attack may effect the use of specific design features, especially those related to shock cancellation off the body.

Table 12: LM 1021-01 Top Uncertain Param-

Table 13: LM 1021-01 Top Uncertain Parameter Contribution to PLdB Total Uncertainty eter Contribution to CSEL Total Uncertainty

\begin{tabular}{ccc}
\hline \hline Uncertain Parameter & Euler & Turbulent \\
\hline Mach Number & $1.4 \%$ & $0.2 \%$ \\
Angle of Attack & $39.0 \%$ & $55.1 \%$ \\
Reflection Factor & $33.8 \%$ & $21.9 \%$ \\
Temperature Profile & $1.6 \%$ & $0.7 \%$ \\
Humidity Profile & $22.7 \%$ & $17.9 \%$ \\
Ground Elevation & $1.6 \%$ & $4.3 \%$ \\
All Others & $<1 \%$ & $<1 \%$ \\
\hline \hline
\end{tabular}

\begin{tabular}{ccc}
\hline \hline Uncertain Parameter & Euler & Turbulent \\
\hline Angle of Attack & $57.2 \%$ & $63.9 \%$ \\
Reflection Factor & $38.2 \%$ & $32.0 \%$ \\
Temperature Profile & $1.1 \%$ & $1.3 \%$ \\
Ground Elevation & $2.4 \%$ & $2.9 \%$ \\
All Others & $<1 \%$ & $<1 \%$ \\
\hline \hline
\end{tabular}

\section{Conclusions}

The objective of this work was to develop and demonstrate a non-intrusive, computationally efficient approach to uncertainty quantification and certification prediction for low-boom, supersonic aircraft configurations. Key components of the developed framework included the use of a surrogate based uncertainty quantification approach using non-intrusive polynomial chaos, the propagation of mixed aleatory and epistemic uncertainties, and a measure of the margin and uncertainty between the design, under uncertainty, and a certification value used for certification prediction. The use of a surrogate based, non-intrusive approach 
was implemented for computationally efficiency and ease of implementation when investigating the largescale, high-fidelity computational fluid dynamics models analyzed in this study. The non-intrusive nature of the approach presented here is advantageous because of its broad applicability to any type of complex, multiphysics simulation.

This process was successfully demonstrated on three low-boom configurations: the Lockheed Martin SEEB-ALR body of revolution, the NASA $69^{\circ}$ Delta Wing, and the Lockheed Martin 1021-01 configuration. Sources of uncertainty considered in the high-fidelity, multiphysics models included variability in freestream conditions, atmospheric profiles, and other modeling parameters used in the propagation of the sonic boom signatures from the near-field to the ground level. Sensitivity analyses were also performed to identify important uncertain parameters. Additionally, the use of an inviscid versus a fully turbulent flow assumption was investigated for each configuration.

The uncertain inputs and subsequent results given in this study were only the finalized results. There were multiple intermediate steps before finalizing uncertain inputs and even the numerical models. One of the key advantages of the process and methodologies outlined in this study, is its applicability in efficiently identifying significant sources of uncertainty and their effect on output uncertainty. Input parameters that contribute significantly to the uncertainty may be subject of refinement, model improvement, or may help identify incorrect models/parameters. For example, in performing this study there was a significant intermediate change in the numerical approached used to calculate the loudness values from the ground level signature. Initial results indicated that the sBOOM sampling rate input parameter contributed as much as $80 \%$ to the total output uncertainty. More importantly, $95 \%$ confidence interval widths for PLdB and CSEL were as much as an order of magnitude greater than the results reported above. This led to further investigation, which resulted in two changes to the analysis. First, the dependence of the loudness metrics on the number of sBOOM sample points was better quantified and slight refinement was made to the epistemic uncertainty range. Second, the information provided in these intermediate results helped identify an issue within the numerical routine used to integrate ground level signature to obtain the loudness metrics. Correcting this issue led to the results detailed throughout this study.

\section{References}

\footnotetext{
${ }^{1}$ Pawlowski, J., Graham, D., Boccadoro, C., Coen, P., and Maglieri, D., "Origins and Overview of the Shaped Sonic Boom Demonstration Program," $43^{\text {rd }}$ AIAA Aerospace Sciences Meeting and Exhibit, Aerospace Sciences Meetings, American Institute of Aeronautics and Astronautics, Jan. 2005.

${ }^{2}$ Howe, D. C., Waithe, K. A., and Haering Jr, E. A., "Quiet SpikeTM Near Field Flight Test Pressure Measurements with Computational Fluid Dynamics Comparisons," $46^{\text {th }}$ AIAA Aerospace Sciences Meeting and Exhibit, AIAA 2008-0128, Jan. 2008.

${ }^{3}$ Morgenstern, J., Norstrud, N., Sokhey, J., Martens, S., and Alonso, J. J., "Advanced Concept Studies for Supersonic Commercial Transports Entering Service in the 2018 to 2020 Period," NASA CR-2013-217820, NASA Langley Research Center, Feb. 2013.

${ }^{4}$ Magee, T. E., Wilcox, P. A., Fugal, S. R., Acheson, K. E., Adamson, E. E., Bidwell, A. L., and Shaw, S. G., "SystemLevel Experimental Validations for Supersonic Commercial Transport Aircraft Entering Service in the 2018-2020 Time Period," NASA CR-2013-217797, NASA Langley Research Center, Feb. 2013.

${ }^{5}$ Yoshida, K., "Supersonic drag reduction technology in the scaled supersonic experimental airplane project by JAXA," Progress in Aerospace Sciences, Vol. 45, No. 4, 2009, pp. 124-146.

${ }^{6}$ Park, M. A., Aftosmis, M. J., Campbell, R. L., Carter, M. B., Cliff, S. E., and Bangert, L. S., "Summary of the 2008 NASA Fundamental Aeronautics Program Sonic Boom Prediction Workshop," Journal of Aircraft, 2014.

${ }^{7}$ Aftosmis, M., Nemec, M., and Cliff, S., "Adjoint-based low-boom design with Cart3D," 29th AIAA Applied Aerodynamics Conference, AIAA 2011-3500, June 2011.

${ }^{8}$ Nemec, M. and Aftosmis, M. J., "Parallel adjoint framework for aerodynamic shape optimization of component-based geometry," $49^{\text {th }}$ AIAA Aerospace Sciences Meeting, AIAA 2011-1249, 2011.

${ }^{9}$ Rallabhandi, S., Nielsen, E., and Diskin, B., "Sonic-Boom Mitigation Through Aircraft Design and Adjoint Methodology," Journal of Aircraft, Vol. 51, No. 2, March-April 2014, pp. 502-510.

${ }^{10}$ Ordaz, I. and Li, W., "Adaptive Aft Signature Shaping of a Low-Boom Supersonic Aircraft Using Off-Body Pressures," $50^{\text {th }}$ AIAA Aerospace Sciences Meeting, AIAA 2012-0020, Jan. 2012.

${ }^{11}$ Biedron, R. T. and et al., "FUN3D Manual: 12.4-69883," Tech. rep., NASA/TM-2014-218179, March 2014.

${ }^{12}$ Park, M. A., "Low Boom Configuration Analysis with FUN3D Adjoint Simulation Framework," 29th AIAA Applied Aerodynamics Conference, AIAA 2011-3337, June 2011.

${ }^{13}$ Park, M. A., Cambell, R. L., Elmiligui, A., Cliff, S. E., and Nayani, S. N., "Specialized CFD Grid Generation Methods for Near-Field Sonic Boom Prediction," 52 ${ }^{\text {nd }}$ AIAA Aerospace Sciences Meeting, AIAA 2014-0115, National Harbor, MD, Jan. 2014.

${ }^{14}$ Casper, J. H., Cliff, S. E., Thomas, S. D., Park, M. A., McMullen, M. S., Melton, J. E., and Durston, D. A., "Assessment
} 
of Near-Field Sonic Boom Simulation Tools," 26 $6^{\text {th }}$ AIAA Applied Aerodynamics Conference, AIAA 2008-6592, Honolulu, HI, Aug. 2008.

${ }^{15}$ Spalart, P. R. and Allmaras, S. R., "One-Equation Turbulence Model for Aerodynamic Flows," 30 ${ }^{\text {th }}$ AIAA Aerospace Sciences Meeting, AIAA 92-1992, Reno, NV, Jan. 1992.

${ }^{16}$ Hunton, L. W., Hicks, R. M., and Mendoza, J. P., "Some Effects of Wing Planform on Sonic Boom," Tech. rep., NASA TN D-7160, Jan. 1973.

${ }^{17}$ Morgenstern, J. M., Buonanno, M., and Marconi, F., "Full Configuration Low Boom Model and Grids for 2014 Sonic Boom Prediction Workshop," 51 ${ }^{\text {st }}$ AIAA Aerospace Sciences Meeting, AIAA 2013-0647, Grapvine, TX, Jan. 2013.

${ }^{18}$ Aftosmis, M. J. and Nemec, M., "Cart3D Simulations for the First AIAA Sonic Boom Prediction Workshop," 52 ${ }^{\text {th }}$ AIAA Aerospace Sciences Meeting, AIAA Paper 2014-0558, Jan. 2014.

${ }^{19}$ Jones, W. T., "GridEx - An Integrated Grid Generation Package for CFD," $16^{\text {th }}$ AIAA Computational Fluid Dynamics Conference, AIAA 2003-4129, Orlando, FL, Aug. 2003.

${ }^{20}$ Rallabhandi, S. K., "Advanced Sonic Boom Prediction Using the Augmented Burgers Equation," Journal of Aircraft, Vol. 48, No. 4, July-Aug. 2011, pp. 1245-1253.

${ }^{21}$ Hosder, S. and Bettis, B., "Uncertainty and sensitivity analysis for reentry flows with inherent and model-form uncertainties," Journal of Spacecraft and Rockets, Vol. 49, No. 2, 2012, pp. 193-206.

${ }^{22}$ Bettis, B., Hosder, S., and Winter, T., "Efficient Uncertainty Quantification in Multidisciplinary Analysis of a Reusable Launch Vehicle," $17^{\text {th }}$ AIAA International Space Planes and Hypersonic Systems and Technologies Conference, AIAA 20112393, April 2011.

${ }^{23}$ Hosder, S., Walters, R. W., and Balch, M., "Point-Collocation Nonintrusive Polynomial Chaos Method for Stochastic Computational Fluid Dynamics," AIAA Journal, Vol. 48, No. 12, 2010, pp. 2721-2730.

${ }^{24}$ Witteveen, J. A. S. and Bijl, H., "Efficient Quantification of the Effect of Uncertainties in AdvectionDiffusion Problems Using Polynomial Chaos," Numerical Heat Transfer, Vol. 53, No. 5, 2008, pp. 437-465.

${ }^{25}$ Han, D. and Hosder, S., "Inherent and Model-Form Uncertainty Analysis for CFD Simulation of Synthetic Jet Actuators," $48^{\text {th }}$ AIAA Aerospace Sciences Meeting, AIAA 2012-0082, Nashville, TN, Jan. 2012.

${ }^{26}$ Eldred, M. S., "Recent Advances in Non-Intrusive Polynomial Chaos and Stochastic Collocation Methods for Uncertainty Analysis and Design," 50 $0^{\text {th }}$ AIAA/ASME/ASCE/AHS/ASC Structures, AIAA 2009-2274, Palm Springs, CA, May 2009.

${ }^{27}$ Walters, R. W. and Huyse, L., "Uncertainty Analysis for Fluid Mechanics with Applications," Tech. rep., ICASE 2002-1, NASA/CR-2002-211449, NASA Langley Research Center, Hampton, VA, 2002.

${ }^{28}$ Ghanem, R. G. and Spanos, P. D., Stochastic Finite Elements: A Spectral Approach, Springer-Verlag, New York, 1991.

${ }^{29}$ West IV, T. K., Hosder, S., and Johnston, C. O., "A Multi-Step Uncertainty Quantification Approach Applied to Hypersonic Reentry Flows," 51 st AIAA Aerospace Sciences Meeting, AIAA 2011-2524, Grapevine, TX, Jan. 2013.

${ }^{30}$ Lockwood, B. and Mavriplis, D., "Gradient-Based Methods for Uncertainty Quantification in Hypersonic Flows," Computers and Fluids Journal, Vol. 85, Oct. 2013, pp. 27-38.

${ }^{31}$ Roderick, O., Anitescu, M., and Fischer, P., "Polynomial Regression Approaches Using Derivative Information for Uncertainty Quantification," Nuclear Science and Engineering, Vol. 164, No. 2, 2010, pp. 122-139.

${ }^{32}$ Hosder, S., Walters, R. W., and Balch, M., "Efficient Sampling for Non-Intrusive Polynomial Chaos Applications with Multiple Uncertain Input Variables," $48^{\text {th }}$ AIAA/ASME/ASCE/AHS/ASC Structures, Structural Dynamics, and Materials Conference, AIAA 2007-0125, Honolulu, HI, 2007.

${ }^{33}$ Oberkampf, W., Helton, J., and Sentz, K., "Mathematical representation of uncertainty," $19^{\text {th }}$ AIAA Applied Aerodynamics Conference, AIAA 2001-1645, June 2001.

${ }^{34}$ Eldred, M. and Swiler, L., "Efficient Algorithms for Mixed Aleatory-Epistemic Uncertainty Quantification with Application to Radiation-Hardened Electronics; Part I: Algorithms and Benchmark Results," Tech. Rep. SAND2009-5805, Sandia National Laboratories, September 2009.

${ }^{35}$ NIST/SEMATECH e-Handbook of Statistical Methods, 2013.

${ }^{36}$ West IV, T. K., Hosder, S., and Winter, T., "Quantification of Margins and Uncertainties for Aerospace Systems using Stochastic Expansions," 52 ${ }^{\text {nd }}$ AIAA Aerospace Sciences Meeting, AIAA 2014-0682, National Harbor, MD, Jan. 2014.

${ }^{37}$ Cliff, S. E., Durston, D. A., Elmiligui, A. A., Jensen, J. C., and Chan, W. M., "Computational and Experimental Assessment of Models for the First AIAA Sonic Boom Prediction Workshop," $52^{\text {nd }}$ AIAA Aerospace Sciences Meeting, AIAA 2014-0560, National Harbor, MD, Jan. 2014.

${ }^{38}$ Sudret, B., "Global sensitivity analysis using polynomial chaos expansion," Reliability Engineering and System Safety, Vol. 93, No. 7, 2008, pp. 964-979.

${ }^{39}$ Crestaux, T., Maitre, O. L., and Martinez, J.-M., "Polynomial chaos expansion for sensitivity analysis," Reliability Engineering and System Safety, 2009.

${ }^{40}$ Ghaffari, S., Magin, T., and Iaccarino, G., "Uncertainty Quantification of Radiative Heat Flux Modeling for Titan Atmospheric Entry," 48 $8^{\text {th }}$ AIAA Aerospace Sciences Meeting, AIAA 2010-0239, Orlando, FL, Jan. 2010.

${ }^{41}$ Loubeau, A. and Coulouvrat, F., "Effects of Meteorological Variability on Sonic Boom Propagation from Hypersonic Aircraft," AIAA Journal, Vol. 47, No. 11, Nov. 2009, pp. 2632-2641. 